\title{
Ordered ground states of kagome magnets with generic exchange anisotropy
}

\author{
Owen Benton 10 \\ RIKEN Center for Emergent Matter Science (CEMS), Wako, Saitama, 351-0198, Japan \\ and Max Planck Institute for the Physics of Complex Systems, Nöthnitzer Str. 38, Dresden 01187, Germany
}

(Received 2 November 2020; accepted 30 April 2021; published 21 May 2021)

\begin{abstract}
There is a growing family of rare-earth kagome materials with dominant nearest-neighbor interactions and strong spin-orbit coupling. The low symmetry of these materials makes theoretical description complicated, with six distinct nearest-neighbor coupling parameters allowed. In this article, we ask what kinds of classical, ordered, ground states can be expected to occur in these materials, assuming generic (i.e., non-fine-tuned) sets of exchange parameters. We use symmetry analysis to show that there are only five distinct classical ground state phases occurring for generic parameters. The five phases are: (i) a coplanar, twofold degenerate state with vanishing magnetization $\left(A_{1}\right)$, (ii) a noncoplanar, twofold degenerate state with magnetization perpendicular to the kagome plane $\left(A_{2}\right)$, (iii) a coplanar, sixfold degenerate state with magnetization lying within the kagome plane (E-coplanar), (iv) a noncoplanar, sixfold degenerate state with magnetization lying within a mirror plane of the lattice (E-noncoplanar ${ }_{6}$ ), (v) a noncoplanar, twelvefold degenerate state with magnetization in an arbitrary direction $\left(E\right.$-noncoplanar $\left.{ }_{12}\right)$. All five are translation invariant $(\mathbf{q}=0)$ states. Having found the set of possible ground states, the ground state phase diagram is obtained by comparing numerically optimized energies for each possibility as a function of the coupling parameters. The state E-noncoplanar ${ }_{12}$ is extremely rare, occupying $\triangleleft \%$ of the full phase diagram, so for practical purposes there are four main ordered states likely to occur in anisotropic kagome magnets with dominant nearest neighbor interactions. These results can aid in interpreting recent experiments on "tripod kagome" systems $\mathrm{R}_{3} \mathrm{~A}_{2} \mathrm{Sb}_{3} \mathrm{O}_{14}$, as well as materials closer to the isotropic limit such as $\mathrm{Cr}$ and $\mathrm{Fe}$ jarosites.
\end{abstract}

DOI: 10.1103/PhysRevB.103.174425

\section{INTRODUCTION}

Frustration can come from various sources. This is certainly true of the frustration exhibited by many magnetic materials, which may be generated by the geometry of the lattice $[1,2]$, by competition between interactions of different kinds $[3,4]$, or by bond-dependent anisotropies $[5,6]$. Sometimes, all of these sources of frustration are present at once, making the problem of determining a ground state both more challenging and more rich [7-9].

Kagome lattice rare-earth materials [10-20] provide a realization of this scenario. The kagome lattice [Fig. 1(a)] is paradigmatic of geometrical frustration while the strong spin-orbit coupling inherent to many rare-earth ions produces complicated anisotropic exchange interactions with distinct, competing contributions and bond dependence.

In this paper we study a model of anisotropic exchange on the kagome lattice, including all possible nearest neighbor interactions consistent with the lattice symmetries [8]. This model has six independent coupling parameters, once one

Published by the American Physical Society under the terms of the Creative Commons Attribution 4.0 International license. Further distribution of this work must maintain attribution to the author(s) and the published article's title, journal citation, and DOI. Open access publication funded by the Max Planck Society. allows for the absence of reflection symmetry in the kagome plane, as is appropriate for many materials.

Several previous works have investigated different types of allowed anisotropic nearest-neighbor interaction on the kagome lattice [8,21-30], but none has treated all possible interactions at once, in the absence of reflection symmetry in the plane. Thus, in some sense, these previous works can be viewed as higher-symmetry limits of the generic case studied here. Our goal in this work is to identify the ordered, classical ground states which are stable over a finite fraction of the six-dimensional parameter space of the full model. We will not address the physics at the phase boundaries between different states or limits featuring high symmetry beyond time reversal and lattice symmetries, or cases of accidental degeneracy, although these can be of interest. In this sense, we are studying those ground states stable in the presence of "generic" exchange anisotropy.

We find that in the full six-dimensional parameter space there are only five such distinct ground states. They are all translationally invariant and may be classified by how they transform under the $C_{3 v}$ point group symmetries of the kagome lattice. Example spin configurations for each are shown in Figs. 2-6.

In addition to materials with strong exchange anisotropy, our approach is also useful for understanding materials where anisotropy is weak but nevertheless plays a key role in selecting the ground state due to the frustrated nature of Heisenberg interactions on the kagome lattice. Our results can be viewed 


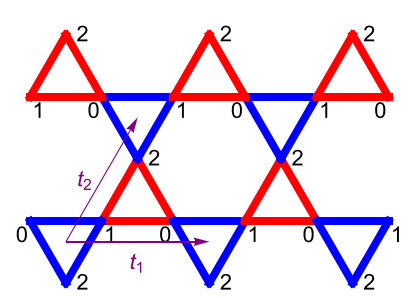

(a)

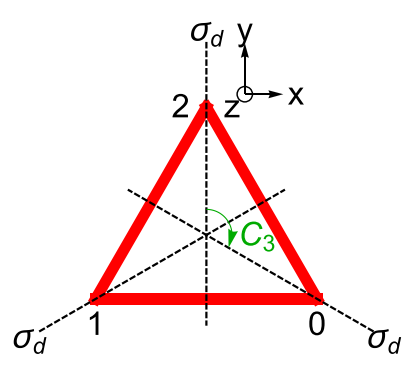

(b)
FIG. 1. (a) The kagome lattice, a network of corner sharing triangles. The labels 0,1,2 indicate the convention used to label the three sublattices of the kagome lattice in this work. $\mathbf{t}_{1}$ and $\mathbf{t}_{2}$ are the basic translations under which the lattice is symmetric (b) The $C_{3 v}$ point group, composed of three reflection symmetries and a threefold rotation axis through the center of the triangle.

as illuminating the spectrum of possible ground states which can be obtained by perturbing an isotropic kagome magnet with various allowed forms of nearest-neighbor exchange anisotropy. This may be of use in understanding the ordered ground states of materials including the $\mathrm{Cr}$ and $\mathrm{Fe}$ jarosites [31-35] and Cd kapellasite [36].

The remainder of this paper is organized as follows:

(i) In Sec. II we review the most general symmetry allowed nearest neighbor exchange Hamiltonian for the kagome lattice $[8,35]$. We then analyze it in terms of the irreducible representations of the point group $C_{3 v}$.

(ii) Building on this symmetry analysis, in Sec. III, we demonstrate the five forms of magnetic order which may arise from the generic Hamiltonian.

(iii) In Sec. IV we use numerical calculations to calculate the ground state phase diagram of the generic Hamiltonian, delineating the regions of parameter space covered by each of the five ordered phases.

(iv) In Sec. V we discuss experimental results on kagome materials in light of our calculations.

(v) In Sec. VI we close with a brief summary and discussion of open directions for future work.



FIG. 2. $A_{1}$ ordered state, occurring as the ground state of Eq. (1) when $\lambda_{A_{1}}<\omega_{A_{2} 0}, \omega_{E 0}$ [Eq. (24)]. All spins lie in the kagome plane at an angle of $\frac{2 \pi}{3}$ to one another and perpendicular to the line joining the spin to the centers of the two neighboring triangles. The spin configuration has vanishing total magnetization, twofold degeneracy, and preserves the lattice symmetries of Eq. (1) while breaking time reversal.

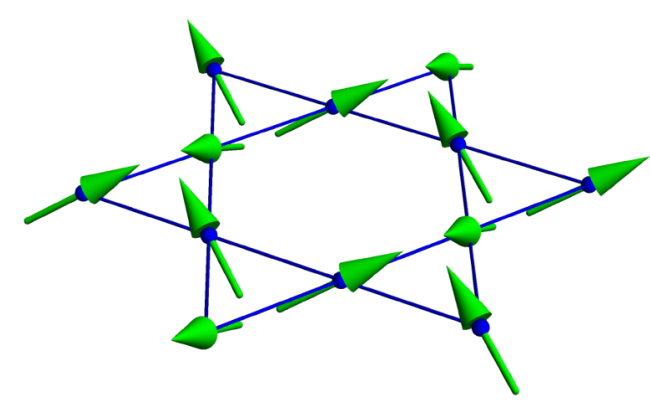

FIG. 3. $A_{2}$ ordered state, occurring as the ground state of Eq. (1) when $\omega_{\mathrm{A}_{2} 0}<\lambda_{\mathrm{A}_{1}}, \omega_{\mathrm{E} 0}$ [Eq. (24)]. The spin configuration has magnetization perpendicular to the kagome plane, twofold degeneracy, and breaks the reflection and time reversal symmetries of Eq. (1).

\section{HAMILTONIAN AND SYMMETRY ANALYSIS}

We consider generalized bilinear anisotropic exchange interactions on a kagome lattice [Fig. 1(a)],

$$
\mathcal{H}=\sum_{\langle i j\rangle} \mathbf{S}_{i} \cdot \mathbf{J}_{i j} \cdot \mathbf{S}_{j}
$$

We require that the interactions respect the following symmetries:

(i) Time reversal

(ii) Lattice translations, $\mathbf{t}_{1}, \mathbf{t}_{2}$, indicated in Fig. 1(a)

(iii) Spatial inversion through lattice sites

(iv) $C_{3}$ rotations around the center of each kagome triangle [Fig. 1(b)]

(v) Reflections in the mirror planes indicated in Fig. 1(b)

We do not assume any reflection symmetry in the plane of the lattice itself.

We assume that the spins $\mathbf{S}_{i}$ transform like magnetic moments, i.e., as axial vectors, odd under time reversal symmetry.

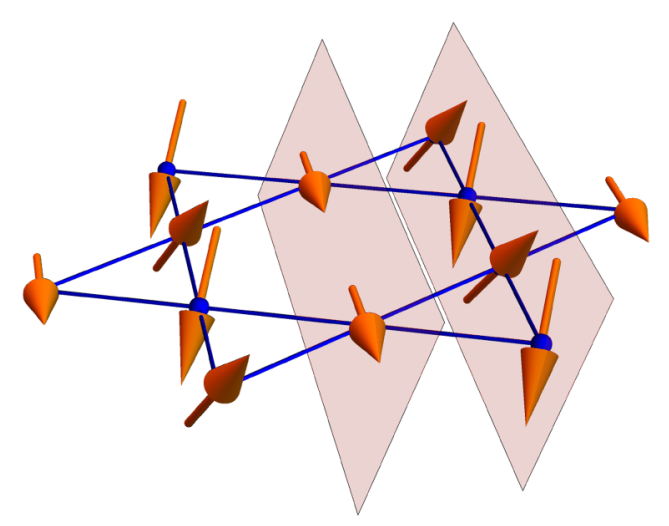

FIG. 4. E-coplanar ordered state. This occurs as a ground state of Eq. (1) in part of the region where $\omega_{\mathrm{E} 0}<\lambda_{\mathrm{A}_{1}}, \omega_{\mathrm{A}_{2} 0}$ [Eq. (24)]. There is one spin lying in the kagome plane and two canted out of it in such a way that the three spins remain coplanar, with the plane of coplanarity being tilted with respect to the kagome plane. The plane of coplanarity is indicated by the translucent red planes. There is a net magnetization within the kagome plane. This state breaks time reversal and all of the point group symmetries of the Hamiltonian, apart from a single reflection symmetry which is preserved. It is sixfold degenerate. 


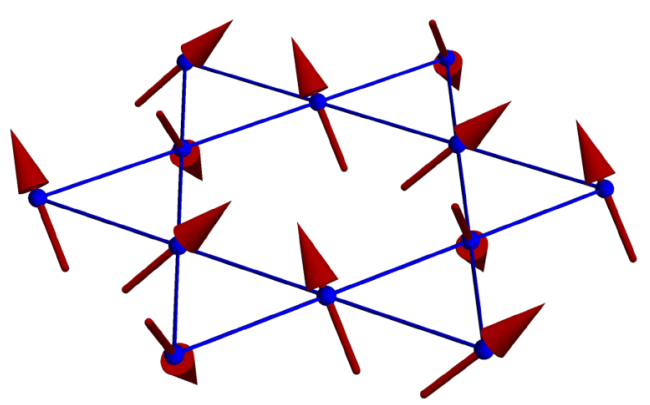

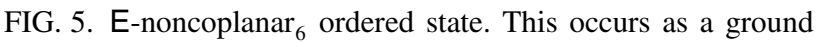
state of Eq. (1) in part of the region where $\omega_{\mathrm{E} 0}<\lambda_{\mathrm{A}_{1}}, \omega_{\mathrm{A}_{2} 0}$ [Eq. (24)]. All spins are canted out of the kagome plane and there is a net magnetization lying within one of the mirror planes of the lattice. This state is noncoplanar and thus has nonzero scalar spin chirality. This state breaks time reversal and all of the point group symmetries of the Hamiltonian but is symmetric under the combination of time reversal and one reflection symmetry. It is sixfold degenerate.

This will apply not only when $\mathbf{S}_{i}$ is a true magnetic moment but also when it is a pseudospin-1/2 degree of freedom describing the twofold degenerate crystal electric field (CEF) ground states of a Kramers ion. In this case the actual magnetic moment is related to the pseudospin via the $\mathrm{g}$ tensor

$$
\mathbf{m}_{i}=\mathbf{g}_{i} \cdot \mathbf{S}_{i} .
$$

An alternative case is possible in which $\mathbf{S}_{i}$ is a pseudospin describing the low energy CEF states of a non-Kramers ion, which will generally be nondegenerate due to a lack of protection from time reversal symmetry. In the non-Kramers case, the pseudospin operators $\mathbf{S}_{i}$ will transform differently under time reversal and the discussion in this section will not apply [37,38].

We now proceed to constrain the form of the exchange matrices $\mathbf{J}_{i j}$ using the symmetries listed above. Time reversal symmetry $\mathcal{T}$

$$
\mathcal{T} \mathbf{S}_{i}=-\mathbf{S}_{i}
$$

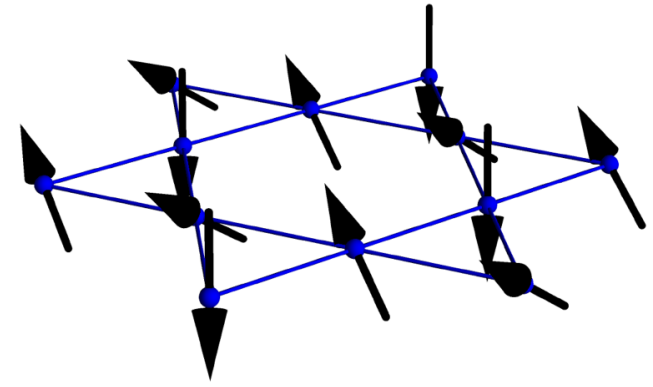


state of Eq. (1) in part of the region where $\omega_{\mathrm{E} 0}<\lambda_{\mathrm{A}_{1}}, \omega_{\mathrm{A}_{2} 0}$ [Eq. (24)]. This state is generally noncoplanar and breaks time reversal symmetry, all point group symmetries, and all combinations of time reversal with point group symmetries. It is twelvefold degenerate. This very low symmetry configuration is rare on the ground state phase diagram, occupying $<1 \%$ of the full parameter space of the Hamiltonian (Fig. 11).

is guaranteed by the bilinear form of Eq. (1).

There are three spins in the unit cell, which we label $\mathbf{S}_{0}, \mathbf{S}_{1}, \mathbf{S}_{2}$ according to the convention in Fig. 1(b). Translational symmetry imposes that the coupling matrices $\mathbf{J}_{i j}$ may only depend on which sublattices $i$ and $j$ belong to and whether the bond $i j$ is on an 'up' or 'down' triangle [red or blue triangles in Fig. 1(a)]. Inversion symmetry then guarantees that 'up' and 'down' triangles have the same coupling matrices. There are thus three different coupling matrices $\mathbf{J}_{01}, \mathbf{J}_{12}, \mathbf{J}_{20}$ entering Eq. (1) which define the interactions between nearest neighbor spins on each pair of sublattices.

The form of the matrices $\mathbf{J}_{i j}$ is constrained by the $C_{3 v}$ point group symmetry at the center of each triangle [Fig. 1(b)] and was given in Refs. [8,35]:

$$
\mathbf{J}_{01}=\left(\begin{array}{ccc}
J_{x} & D_{z} & -D_{y} \\
-D_{z} & J_{y} & K \\
D_{y} & K & J_{z}
\end{array}\right)
$$

There are six independent parameters in these exchange matrices: Three diagonal exchanges $J_{x}, J_{y}, J_{z}$, two DzyaloshinskiiMoriya (DM) interactions $D_{y}, D_{z}$, and one symmetric offdiagonal exchange $K$.

An additional symmetry which could, in principle, be present is reflection symmetry in the plane of the kagome lattice. The presence of such a symmetry would reduce the set of allowed exchange parameters to four, by setting $D_{y}=K=0$. This case was discussed in detail in Ref. [8]. In this work, we will continue to assume that there is no reflection symmetry in the kagome plane, as is appropriate for many rare-earth kagome materials [17]. Therefore, we shall take both $D_{y}$ and $K$ to be nonzero.

To begin in determining the phase diagram it is helpful to rewrite the Hamiltonian in terms of objects $\mathbf{m}_{\gamma, k}$ transforming according to the irreducible representations (irreps) of the point group. $\mathbf{m}_{\gamma, k}$ are defined for each triangle of the lattice, which we index using $k$. This approach is discussed 
for the kagome lattice in Ref. [8] and the pyrochlore lattice in Ref. [7].

These objects can function as local order parameters for the different kinds of three-sublattice order which we will encounter on the phase diagram of the anisotropic exchange model. They also aid in the determination of the phase diagram itself. The appropriate objects are defined in Ref. [8] but we reintroduce them here since they are essential to our discussion.

Firstly, there is one scalar object transforming according to the trivial $A_{1}$ representation of $C_{3 v}$. A nonzero average value of this field breaks none of the point-group symmetries, only breaking time-reversal symmetry.

$m_{\mathrm{A}_{1}, k}=\frac{1}{3}\left(\frac{1}{2} S_{0, k}^{x}+\frac{\sqrt{3}}{2} S_{0, k}^{y}+\frac{1}{2} S_{1, k}^{x}-\frac{\sqrt{3}}{2} S_{1, k}^{y}-S_{2, k}^{x}\right)$
Here $S_{i, k}^{\alpha}$ is the $\alpha$ component of the spin belonging to sublattice $i$ and triangle $k$.

There are then two linearly independent scalars, which transform according to the $A_{2}$ representation. A nonzero average value of these fields breaks time reversal symmetry and all three mirror symmetries of $C_{3 v}$ but preserves the threefold rotational symmetry.

$$
\begin{gathered}
m_{\mathrm{A}_{2} \mathrm{a}, k}=\frac{1}{3}\left(S_{0, k}^{z}+S_{1, k}^{z}+S_{2, k}^{z}\right) \\
m_{\mathrm{A}_{2} \mathrm{~b}, k}=\frac{1}{3}\left(-\frac{\sqrt{3}}{2} S_{0, k}^{x}+\frac{1}{2} S_{0, k}^{y}+\frac{\sqrt{3}}{2} S_{1, k}^{x}+\frac{1}{2} S_{1, k}^{y}-S_{2, k}^{y}\right)
\end{gathered}
$$

Finally, there are three two-component vectors, transforming according to the two-dimensional E-irrep of $C_{3 v}$

$$
\begin{gathered}
\mathbf{m}_{\mathrm{Ea}, k}=\frac{1}{3}\left(\begin{array}{c}
S_{0, k}^{x}+S_{1, k}^{x}+S_{2, k}^{x} \\
S_{0, k}^{y}+S_{1, k}^{y}+S_{2, k}^{y}
\end{array}\right) \\
\mathbf{m}_{\mathrm{Eb}, k}=\frac{1}{3}\left(\begin{array}{c}
\frac{1}{2} S_{0, k}^{x}-\frac{\sqrt{3}}{2} S_{0, k}^{y}+\frac{1}{2} S_{1, k}^{x}+\frac{\sqrt{3}}{2} S_{1, k}^{y}-S_{2, k}^{x} \\
-\frac{\sqrt{3}}{2} S_{0, k}^{x}-\frac{1}{2} S_{0, k}^{y}+\frac{\sqrt{3}}{2} S_{1, k}^{x}-\frac{1}{2} S_{1, k}^{y}+S_{2, k}^{y}
\end{array}\right) \\
\mathbf{m}_{\mathrm{Ec}, k}=\frac{1}{3}\left(\begin{array}{c}
\sqrt{\frac{3}{2}}\left(S_{0, k}^{z}-S_{1, k}^{z}\right) \\
\sqrt{2}\left(-\frac{1}{2} S_{0, k}^{z}-\frac{1}{2} S_{1, k}^{z}+S_{2, k}^{z}\right)
\end{array}\right)
\end{gathered}
$$

In terms of these objects the Hamiltonian may be written

$$
\begin{aligned}
\mathcal{H}= & \frac{3}{2} \sum_{k}\left[\lambda_{\mathrm{A}_{1}} m_{\mathrm{A}_{1}, k}^{2}+\left(m_{\mathrm{A}_{2} \mathrm{a}, k}, m_{\mathrm{A}_{2} \mathrm{~b}, k}\right)\left(\begin{array}{cc}
\lambda_{\mathrm{A}_{2}, \mathrm{aa}} & \frac{\lambda_{\mathrm{A}_{2}, \mathrm{ab}}}{2} \\
\frac{\lambda_{\mathrm{A}_{2}, \mathrm{ab}}}{2} & \lambda_{\mathrm{A}_{2}, \mathrm{bb}}
\end{array}\right)\left(\begin{array}{l}
m_{\mathrm{A}_{2} \mathrm{a}, k} \\
m_{\mathrm{A}_{2} \mathrm{~b}, k}
\end{array}\right)\right. \\
& \left.+\left(\mathbf{m}_{\mathrm{Ea}, k}, \mathbf{m}_{\mathrm{Eb}, k}, \mathbf{m}_{\mathrm{Ec}, k}\right)\left(\begin{array}{ccc}
\lambda_{\mathrm{E}, \mathrm{aa}} & \frac{\lambda_{\mathrm{E}, \mathrm{ab}}}{2} & \frac{\lambda_{\mathrm{E}, \mathrm{ac}}}{2} \\
\frac{\lambda_{\mathrm{E}, \mathrm{ab}}}{2} & \lambda_{\mathrm{E}, \mathrm{bb}} & \frac{\lambda_{\mathrm{E}, \mathrm{bc}}}{2} \\
\frac{\lambda_{\mathrm{E}, \mathrm{ac}}}{2} & \frac{\lambda_{\mathrm{E}, \mathrm{bc}}}{2} & \lambda_{\mathrm{E}, \mathrm{cc}}
\end{array}\right)\left(\begin{array}{l}
\mathbf{m}_{\mathrm{Ea}, k} \\
\mathbf{m}_{\mathrm{Eb}, k} \\
\mathbf{m}_{\mathrm{Ec}, k}
\end{array}\right)\right] \\
= & \frac{3}{2} \sum_{k}\left[\lambda_{\mathrm{A}_{1}} m_{\mathrm{A}_{1}, k}^{2}+\left(m_{\mathrm{A}_{2} \mathrm{a}, k}, m_{\mathrm{A}_{2} \mathrm{~b}, k}\right) \Lambda_{\mathrm{A}_{2}}\left(\begin{array}{l}
m_{\mathrm{A}_{2} \mathrm{a}, k} \\
m_{\mathrm{A}_{2} \mathrm{~b}, k}
\end{array}\right)+\left(\mathbf{m}_{\mathrm{Ea}, k}, \mathbf{m}_{\mathrm{Eb}, k}, \mathbf{m}_{\mathrm{Ec}, k}\right) \Lambda_{\mathrm{E}}\left(\begin{array}{l}
\mathbf{m}_{\mathrm{Ea}, k} \\
\mathbf{m}_{\mathrm{Eb}, k} \\
\mathbf{m}_{\mathrm{Ec}, k}
\end{array}\right)\right]
\end{aligned}
$$

where $k$ indexes the triangles of the lattice and the final term in Eq. (13) should be interpreted as a sum of nine scalar products between the vectors $\mathbf{m}_{\mathrm{Ei}, k}$. The coefficients $\lambda_{\gamma}$ are.

$$
\begin{gathered}
\lambda_{\mathrm{A}_{1}}=\frac{1}{2}\left(-2 \sqrt{3} D_{z}+J_{x}-3 J_{y}\right) \\
\lambda_{\mathrm{A}_{2}, \text { aa }}=2 J_{z} \\
\lambda_{\mathrm{A}_{2}, \mathrm{bb}}=\frac{1}{2}\left(-2 \sqrt{3} D_{z}-3 J_{x}+J_{y}\right)
\end{gathered}
$$

$$
\begin{gathered}
\lambda_{\mathrm{A}_{2}, \mathrm{ab}}=2\left(\sqrt{3} D_{y}+K\right) \\
\lambda_{\mathrm{E}, \mathrm{aa}}=J_{x}+J_{y} \\
\lambda_{\mathrm{E}, \mathrm{bb}}=\sqrt{3} D_{z}-\frac{J_{x}}{2}-\frac{J_{y}}{2} \\
\lambda_{\mathrm{E}, \mathrm{cc}}=-J_{z} \\
\lambda_{\mathrm{E}, \mathrm{ab}}=J_{x}-J_{y}
\end{gathered}
$$




$$
\begin{gathered}
\lambda_{\mathrm{E}, \mathrm{ac}}=\sqrt{6} D_{y}-\sqrt{2} K \\
\lambda_{\mathrm{E}, \mathrm{bc}}=\sqrt{8} K .
\end{gathered}
$$

It is then useful to write Eq. (13) in a new basis chosen to diagonalize the matrices $\Lambda_{\mathrm{A}_{2}}$ and $\Lambda_{\mathrm{E}}$.

$$
\begin{aligned}
\mathcal{H}= & \frac{3}{2} \sum_{k}\left(\lambda_{\mathrm{A}_{1}} m_{\mathrm{A}_{1}, k}^{2}+\omega_{\mathrm{A}_{2} 0} m_{\mathrm{A}_{2} 0, k}^{2}+\omega_{\mathrm{A}_{2} 1} m_{\mathrm{A}_{2} 1, k}^{2}\right. \\
& \left.+\omega_{\mathrm{E} 0} \mathbf{m}_{\mathrm{E} 0, k}^{2}+\omega_{\mathrm{E} 1} \mathbf{m}_{\mathrm{E} 1, k}^{2}+\omega_{\mathrm{E} 2} \mathbf{m}_{\mathrm{E} 2, k}^{2}\right)
\end{aligned}
$$

Here $\omega_{\mathrm{A}_{2} i}(i=0,1)$ are the eigenvalues of $\Lambda_{\mathrm{A}_{2}}$ and $m_{\mathrm{A}_{2} i}$ are linear combinations of $m_{\mathrm{A}_{2} \mathrm{a}}$ and $m_{\mathrm{A}_{2} \mathrm{~b}}$ corresponding to the associated eigenvector of $\Lambda_{\mathrm{A}_{2}}$ [(Eq. 13)]. Similarly, $\omega_{\mathrm{E} i}(i=$ $0,1,2)$ are the eigenvalues of $\Lambda_{\mathrm{E}}$ and $\mathbf{m}_{\mathrm{E} i}$ are linear combinations of $\mathbf{m}_{\mathrm{Ea}}, \mathbf{m}_{\mathrm{Eb}}$, and $\mathbf{m}_{\mathrm{Ec}}$ corresponding to the associated eigenvector of $\Lambda_{\mathrm{E}}$. We define, without loss of generality,

$$
\omega_{\mathrm{A}_{2} \mathrm{O}} \leqslant \omega_{\mathrm{A}_{2} 1}, \quad \omega_{\mathrm{E} 0} \leqslant \omega_{\mathrm{E} 1} \leqslant \omega_{\mathrm{E} 2} .
$$

In this work we will treat the spins as classical vectors of fixed length $\left|\mathbf{S}_{i}\right|=1$. Due to this condition, the following constraint applies to fields $\mathbf{m}_{\gamma}$ defined in Eqs. (7)-(12):

$$
\sum_{\gamma}\left|\mathbf{m}_{\gamma, k}\right|^{2}=1, \forall k
$$

Equation (26) is a necessary but not sufficient condition for the proper normalization of the spins.

It should be emphasized that the reformulation of the problem in terms of variables $\mathbf{m}_{\gamma, k}$ does not require any further assumptions beyond the nearest-neighbor bilinear nature of the interactions and the symmetries enumerated at the beginning of this section. In what follows we will seek to find the classical ground states of Eq. (1).

\section{WHAT KINDS OF CLASSICAL GROUND STATE ARE POSSIBLE?}

In this section we seek to establish the possible classical ordered phases which may occur on the ground state phase diagram of Eq. (1). Our focus is on classical ground states which are stable over finite regions of the full six-dimensional parameter space. So, although there may be additional ground states which become relevant in particular high symmetry limits of Eq. (1), these are not the subject of our present discussion as they rely on fine tuning of parameters.

Our conclusions may be summarized as follows:

(1) A translation invariant $(\mathbf{q}=0)$ ground state exists for all values of exchange parameters.

(2) If

$$
\lambda_{\mathrm{A}_{1}}<\omega_{\mathrm{A}_{2} 0}, \omega_{\mathrm{E} 0}
$$

the ground state is the antiferromagnetic state shown in Fig. 2 and discussed in Sec. III B.

(3) If

$$
\omega_{\mathrm{A}_{2} 0}<\lambda_{\mathrm{A}_{1}}, \omega_{\mathrm{E} 0}
$$

the ground state is the noncoplanar state, with magnetization perpendicular to the plane, shown in Fig. 3 and discussed in Sec. III C.
(4) If

$$
\omega_{\mathrm{E} 0}<\lambda_{\mathrm{A}_{2}}, \omega_{A_{1}}
$$

the ground state may be one of three states (E-coplanar, E-noncoplanar ${ }_{6}$, E-noncoplanar ${ }_{12}$ ) shown in Figs. 4-6 and discussed in Sec. III D.

A summary of the five phases in terms of the values of local order parameters $\mathbf{m}_{\gamma}$ [Eqs. (7)-(12)] is given in Table I.

In what follows we will demonstrate these results.

\section{A. Existence of $q=0$ classical ground state for all parameter sets}

Here, for completeness, we give the proof that Eq. (1) possesses a $\mathbf{q}=0$ classical ground state for all values of exchange parameters, following arguments previously given in Refs. [7,8]. We follow a strategy of building up the global ground state from the ground states of corner sharing units, as is frequently done for models on lattices with a corner-sharing structure [7,8,29,30,39].

As we have shown above, the nearest-neighbor exchange Hamiltonian Eq. (1) can be rewritten as a sum over triangles:

$$
\mathcal{H}=\sum_{\Delta} \mathcal{H}_{\triangle}
$$

with $\mathcal{H}_{\triangle}$ being the same on every triangle of the lattice, as a consequence of inversion and translation symmetries. This formulation makes it clear that any configuration which minimizes the energy of each individual triangle also minimizes the energy of the system as a whole.

Such a configuration may readily be obtained by minimizing the energy on a single "up-pointing" triangle [red triangles in Fig. 1(a)] and then tiling the solution over all "up-pointing" triangles of the lattice. The "up-pointing" triangles will then all be in a ground state by construction, and the "downpointing" triangles will be too, because they have the same exchange matrices as "up-pointing" triangles and the same spin orientation on each sublattice.

This naturally results in a translation invariant $(\mathbf{q}=0)$ state, which is guaranteed to be a ground state. Moreover, it means that the ground state problem on the whole lattice can be reduced to finding the ground state of three spins on a triangle.

In Secs. III B-IIID we examine the various possible solutions to this problem that occur in different regions of parameter space. The argument above does not rule out the existence of additional, $\mathbf{q} \neq 0$, ground states, degenerate with the $\mathbf{q}=0$ ones. We regard it, however, as unlikely that such accidental degeneracies are present over finite regions of the six-dimensional parameter space. Such a robust accidental degeneracy would require a pair of states not related by any symmetry to be degenerate with respect to each of the six independent terms of the Hamiltonian individually, which would seem to require a rather large coincidence. The Heisenberg-Kitaev model on the kagome lattice [29,30] exhibits an extended, accidental degeneracy, in the classical limit, but since that model only has two symmetry distinct terms the required coincidence is not so large.

From now on, we assume translationally invariant ground states built by tiling the ground states of a single triangle 
TABLE I. Description of the five possible classical ground states in terms of the local order parameters defined for a triangle in Eqs. (7)(12). E order parameters $\mathbf{m}_{\mathrm{E}_{\alpha}}$ are expressed in polar form, with $m_{\mathrm{E}_{\alpha}}$ and $\psi_{\alpha}$ as defined in Eqs. (39)-(41). $n$ is an integer, with different choices of $n$ corresponding to different degenerate ground states in E-coplanar and E-noncoplanar ${ }_{6}$ phases.

\begin{tabular}{|c|c|c|c|c|c|c|c|c|c|}
\hline $\mathrm{A}_{1}$ & $\neq 0$ & 0 & 0 & 0 & & 0 & & 0 & \\
\hline E-coplanar & $\neq 0$ & 0 & 0 & $\neq 0$ & $\frac{n \pi}{3}$ & $\neq 0$ & $\frac{n \pi}{3}$ & $\neq 0$ & $\frac{n \pi}{3}$ \\
\hline E-noncoplanar 6 & 0 & $\neq 0$ & $\neq 0$ & $\neq 0$ & $\frac{(2 n+1) \pi}{6}$ & $\neq 0$ & $\frac{(2 n+1) \pi}{6}$ & $\neq 0$ & $\frac{(2 n+1) \pi}{6}$ \\
\hline E-noncoplanar ${ }_{12}$ & $\neq 0$ & $\neq 0$ & $\neq 0$ & $\neq 0$ & {$[0, \pi)$} & $\neq 0$ & {$[0, \pi)$} & $\neq 0$ & {$[0, \pi)$} \\
\hline
\end{tabular}

and therefore drop the triangle index $k$ from the fields $\mathbf{m}_{\gamma, k}$ and spins $\mathbf{S}_{i, k}$. We can use the solutions of the single triangle problem to check the validity of the assumption that there are only $\mathbf{q}=0$ ground states. We do this by checking whether two distinct single triangle ground states can be placed on neighboring triangles without causing an inconsistency at the shared site. If they cannot, then only $\mathbf{q}=0$ ground states are possible. This is explicitly checked for each single triangle ground state below, and in each case we find that $\mathbf{q} \neq 0$ states are only possible with fine tuning.

\section{B. $A_{1}$ order}

We first consider the parameter regime defined by inequality (27) where $\lambda_{A_{1}}$ is the lowest coefficient in Eq. (24). We can use Eq. (26) to write:

$$
m_{A_{1}}^{2}=1-\sum_{\gamma \neq A_{1}} m_{\gamma}^{2}
$$

and so eliminate $m_{\mathrm{A}_{1}}$ from the Hamiltonian [Eq. (24)]:

$$
\begin{aligned}
\mathcal{H}= & \frac{3}{2} \sum_{\Delta}\left(\lambda_{\mathrm{A}_{1}}+\left(\omega_{\mathrm{A}_{2} 0}-\lambda_{\mathrm{A}_{1}}\right) m_{\mathrm{A}_{2} 0}^{2}+\left(\omega_{\mathrm{A}_{2} 1}-\lambda_{\mathrm{A}_{1}}\right) m_{\mathrm{A}_{2} 1}^{2}\right. \\
& +\left(\omega_{\mathrm{E} 0}-\lambda_{\mathrm{A}_{1}}\right) \mathbf{m}_{\mathrm{E} 0}^{2}+\left(\omega_{\mathrm{E} 1}-\lambda_{\mathrm{A}_{1}}\right) \mathbf{m}_{\mathrm{E} 1}^{2} \\
& \left.+\left(\omega_{\mathrm{E} 2}-\lambda_{\mathrm{A}_{1}}\right) \mathbf{m}_{\mathrm{E} 2}^{2}\right) .
\end{aligned}
$$

All the remaining fields $m_{\mathrm{A}_{2} \mathrm{i}}, \mathbf{m}_{\mathrm{Ei}}$ now appear as quadratic forms with positive coefficients, due to inequalities (25) and (27).

Therefore any spin configuration where all these fields vanish is necessarily a ground state, for all parameter sets fulfilling the inequality (27). There are exactly two such configurations, related to each other by time reversal symmetry:

$$
\begin{aligned}
& \mathbf{S}_{0}= \pm\left(\frac{1}{2}, \frac{\sqrt{3}}{2}, 0\right), \\
& \mathbf{S}_{1}= \pm\left(\frac{1}{2},-\frac{\sqrt{3}}{2}, 0\right), \\
& \mathbf{S}_{2}= \pm(-1,0,0) .
\end{aligned}
$$

These are the ground state spin configurations of the $A_{1}$ phase. The only remaining degree of freedom on a triangle is the choice of the + or - sign in Eq. (33). Once this sign is chosen for one triangle, consistency at the shared spin requires that the same sign is chosen on the neighboring triangles. Propagating this throughout the lattice we see that only $\mathbf{q}=0$ tilings are possible.
This phase preserves all lattice symmetries of the original Hamiltonian but breaks time reversal symmetry. One of the ground states is illustrated in Fig. 2.

\section{C. $A_{2}$ order}

Next we consider parameter sets falling in the regime described by inequality (28), such that $\omega_{\mathrm{A}_{2} 0}$ is the lowest coefficient in Eq. (24). Under these conditions we can use Eq. (26) to remove $m_{\mathrm{A}_{2} \mathrm{O}}$ from the Hamiltonian [Eq. (24)] in a similar manner to the analysis in Sec. III B. By this means one can show that the ground states for parameter sets obeying the inequality (28) are of the form

$$
\begin{aligned}
& \mathbf{S}_{0}= \pm\left(-\frac{\sqrt{3}}{2} \cos (\eta), \frac{1}{2} \cos (\eta),-\sin (\eta)\right) \\
& \mathbf{S}_{1}= \pm\left(\frac{\sqrt{3}}{2} \cos (\eta), \frac{1}{2} \cos (\eta),-\sin (\eta)\right) \\
& \mathbf{S}_{2}= \pm(0,-\cos (\eta),-\sin (\eta)) .
\end{aligned}
$$

With the out-of-plane canting angle $\eta$ being determined by the content of the lowest eigenvector of $\Lambda_{\mathrm{A}_{2}}$ [Eq. (13)]. In terms of the coupling parameters, $\eta$ obeys the relation:

$$
\tan (2 \eta)=\frac{4\left(\sqrt{3} D_{y}+K\right)}{2 \sqrt{3} D_{z}+3 J_{x}-J_{y}+4 J_{z}} .
$$

With $\eta$ fixed by Eq. (35), the only remaining degree of freedom on a single triangle is the choice of sign in Eq. (34). Once this sign is chosen for one triangle, consistency at the shared spin requires that the same sign is chosen on the neighboring triangles. Propagating this throughout the lattice we see that only $\mathbf{q}=0$ tilings are possible.

The $A_{2}$ configurations have nonzero scalar chirality on the triangle:

$$
\chi=\left(\mathbf{S}_{0} \times \mathbf{S}_{1}\right) \cdot \mathbf{S}_{2}= \pm \frac{3 \sqrt{3}}{2} \cos (\eta)^{2} \sin (\eta)
$$

This phase breaks the reflection and time reversal symmetry of $\mathcal{H}$ but preserves the $C_{3}$ rotational symmetry. An example ground state in this phase is illustrated in Fig. 3.

\section{E orders}

We then come to the case

$$
\omega_{E 0}<\lambda_{A_{1}}, \omega_{\mathrm{A}_{2} 0} \text {. }
$$

Applying the same type of arguments as in Secs. III B and IIIC, we might expect to find a ground state with $m_{\mathrm{A}_{1}}=$ 
$m_{\mathrm{A}_{2} \mathrm{a}}=m_{\mathrm{A}_{2} \mathrm{~b}}=0$ and with the values of $m_{\mathrm{Ea}, \mathrm{b}, \mathrm{c}}$ being determined by the lowest eigenvector of $\Lambda_{\mathrm{E}}$. However, for typical eigenvectors of $\Lambda_{E}$ this is incompatible with the spin length constraints

$$
\mathbf{S}_{0}^{2}=\mathbf{S}_{1}^{2}=\mathbf{S}_{2}^{2}=1 .
$$

The resolution of this is that the system must mix small values of $m_{\mathrm{A}_{1}}, m_{\mathrm{A}_{2} \mathrm{a}}, m_{\mathrm{A}_{2} \mathrm{~b}}$ into the ground state, so as to respect the spin length constraints while retaining a large value of $\left|\mathbf{m}_{\mathrm{E} 0}\right|$ as favored by the Hamiltonian.

We can distinguish the different ways that this can happen by further consideration of the symmetries of the problem. Specifically, we can ask what symmetries of the Hamiltonian can be preserved in the presence of nonzero values of $\mathbf{m}_{\mathrm{Ea}}, \mathbf{m}_{\mathrm{Eb}}, \mathbf{m}_{\mathrm{Ec}}$.

There are three possibilities consistent with nonzero values of $m_{\mathrm{E} \alpha}$.

(1) One of the reflection symmetries of $C_{3 v}$ is preserved. This corresponds to the E-coplanar phase discussed below in Sec. III D 1.

(2) The combination of one of the reflection symmetries of $C_{3 v}$ with time reversal is preserved. This corresponds to the E-noncoplanar ${ }_{6}$ phase discussed below in Sec. III D 2.

(3) None of the point group symmetries nor any of their combinations with time reversal symmetry are preserved. This

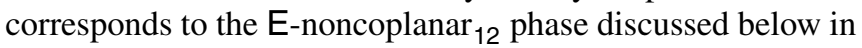
Sec. III D 3.

\section{E-coplanar}

In the E-coplanar phase one of the reflection symmetries of $C_{3 v}$ is preserved. For concreteness, let us suppose that the preserved symmetry is reflection in the $y z$ plane, i.e., the mirror plane that runs through site 2 in Fig. 1(b). We write $\mathbf{m}_{\mathrm{Ea}}, \mathbf{m}_{\mathrm{Eb}}, \mathbf{m}_{\mathrm{Ec}}$ in polar form

$$
\begin{aligned}
& \mathbf{m}_{\mathrm{Ea}}=m_{\mathrm{Ea}}\left(\begin{array}{c}
\cos \left(\psi_{\mathrm{Ea}}\right) \\
\sin \left(\psi_{\mathrm{Ea}}\right)
\end{array}\right) \\
& \mathbf{m}_{\mathrm{Eb}}=m_{\mathrm{Eb}}\left(\begin{array}{c}
\cos \left(\psi_{\mathrm{Eb}}\right) \\
\sin \left(\psi_{\mathrm{Eb}}\right)
\end{array}\right) \\
& \mathbf{m}_{\mathrm{Ec}}=m_{\mathrm{Ec}}\left(\begin{array}{c}
\cos \left(\psi_{\mathrm{Ec}}\right) \\
\sin \left(\psi_{\mathrm{Ec}}\right)
\end{array}\right)
\end{aligned}
$$

defining the angles $\psi_{\mathrm{Ei}}$ to lie in the interval $[0, \pi)$, and allowing the scalars $m_{\mathrm{Ei}}$ to take either sign \pm . Imposing preservation of reflection symmetry in the $y z$ plane constrains $\psi_{\mathrm{E} \alpha}$

$$
\psi_{\mathrm{E} \alpha}=0 \quad \forall \alpha .
$$

More generally, if we had chosen one of the other mirror planes [Fig. 1(b)] to be preserved, we would have $\psi_{\mathrm{E} \alpha}=$ $\frac{n \pi}{3}, n \in\{0,1,2\}$. If the preserved reflection plane passes through site 2 of the unit cell [see Fig. 1(a)] then $n=0$, if through site 0 then $n=1$ and if through site 1 then $n=2$.

The symmetry further implies that

$$
m_{\mathrm{A}_{2} \mathrm{a}}=m_{\mathrm{A}_{2} \mathrm{~b}}=0
$$

but a nonzero value of $m_{\mathrm{A}_{1}}$ is allowed

$$
m_{\mathrm{A}_{1}} \neq 0
$$

and will be mixed into the ground state in such a way as to satisfy the spin length constraints. The magnitudes and relative signs of $m_{\mathrm{Ei}}, m_{\mathrm{A}_{1}}$ are fixed by minimizing the energy.

An example spin configuration on the three sublattices in this phase has the form (taking $n=0$ )

$$
\begin{aligned}
& \mathbf{S}_{0}=(\cos (\phi) \sin (\theta), \sin (\phi) \sin (\theta), \cos (\theta)) \\
& \mathbf{S}_{1}=(\cos (\phi) \sin (\theta),-\sin (\phi) \sin (\theta),-\cos (\theta)) \\
& \mathbf{S}_{2}=(1,0,0),
\end{aligned}
$$

where $\phi$ and $\theta$ are functions of the exchange parameters, which must be determined by minimizing the energy. Degenerate spin configurations can be obtained by applying time reversal and lattice symmetries to Eq. (45) and there is a total degeneracy of six.

The spins are in a common plane, which is generally not the plane of the kagome lattice. The total magnetization of the configuration is normal to the unbroken mirror plane. An example configuration is shown in Fig. 4.

Minimizing the energy with respect to $\theta$ and $\phi$ gives a pair of equations which relate the ground state canting angles to the coupling parameters.

$$
\begin{aligned}
\frac{\partial E}{\partial \theta}= & \\
\Rightarrow & \frac{1}{2} \cos (\theta)\left(\cos (\phi)+4 \cos (\phi)^{2} \sin (\theta)-\sqrt{3} \sin (\phi)\right) J_{x} \\
& +\frac{1}{2} \cos (\theta)(3 \cos (\phi)+\sin (\phi)(\sqrt{3}-4 \sin (\theta) \sin (\phi))) J_{y} \\
& +\sin (2 \theta) J_{z}+(2 \cos (2 \theta) \cos (\phi)-\sin (\theta)) D_{y} \\
& +2 \cos (\theta)(1-2 \cos (\phi) \sin (\theta)) \sin (\phi) D_{z} \\
& +(\sqrt{3} \sin (\theta)-2 \cos (2 \theta) \sin (\phi)) K=0 \\
\frac{\partial E}{\partial \phi}= & 0 \\
\Rightarrow & -\frac{1}{2} \sin (\theta)(\sqrt{3} \cos (\phi)+\sin (\phi)+2 \sin (\theta) \sin (2 \phi)) J_{x} \\
& +\frac{1}{2} \sin (\theta)(\sqrt{3} \cos (\phi)-3 \sin (\phi)-2 \sin (\theta) \sin (2 \phi)) J_{y} \\
& -2 \cos (\theta) \sin (\theta) \sin (\phi) D_{y} \\
& +2 \sin (\theta)(\cos (\phi)-\cos (2 \phi) \sin (\theta)) D_{z} \\
& -2 \cos (\theta) \cos (\phi) \sin (\theta) K=0
\end{aligned}
$$

If the angles $\theta$ and $\phi$ are measured for a given material (e.g., from refinement of Bragg peaks), then Eqs. (46) and (47) can be used to give constraints on the coupling parameters, at least at the level of a classical description.

Unless the angles $\phi, \theta$ are fine tuned to special values (which requires fine tuning of exchange parameters), there is no way to place different members of the set of six singletriangle ground states on neighboring triangles. This implies that only $\mathbf{q}=0$ configurations are possible within this phase, for generic parameters.

\section{E-noncoplanar 6}

In the E-noncoplanar ${ }_{6}$ phase the combination of time reversal with one of the reflection symmetries of $C_{3 v}$ is preserved. For concreteness, let us suppose the preserved symmetry is the combination of time reversal with the mirror plane that runs through site 2 in Fig. 1(b). 
This symmetry constrains the angles $\psi_{\mathrm{E} \alpha}$ [Eqs. (39)-(41)], remembering that $\psi_{\mathrm{E} \alpha}$ is defined to lie in the interval $[0, \pi)$ :

$$
\psi_{\mathrm{E} \alpha}=\pi / 2 \quad \forall \alpha
$$

More generally, if we had chosen one of the other mirror planes [Fig. 1(b)] to be preserved when in combination with $\mathcal{T}$, we would have $\psi_{\mathrm{E} \alpha}=\frac{(2 n+1) \pi}{6}, n \in\{0,1,2\}$. If the mirror plane preserved in combination with $\mathcal{T}$ runs through site 2 of the unit cell [see Fig. 1(a)] then $n=1$, if through site 0 then $n=2$, if through site 1 then $n=0$.

The symmetry implies that

$$
m_{\mathrm{A}_{1}}=0
$$

but nonzero values of $m_{\mathrm{A}_{2} \mathrm{a}}$ and $m_{\mathrm{A}_{2} \mathrm{~b}}$ appear in the ground state as a way to satisfy the spin length constraints

$$
m_{\mathrm{A}_{2}}, m_{\mathrm{A}_{2} \mathrm{~b}} \neq 0
$$

An example spin configuration for this phase is

$$
\begin{aligned}
& \mathbf{S}_{0}=(\cos (v) \sin (\mu), \sin (v) \sin (\mu), \cos (\mu)) \\
& \mathbf{S}_{1}=(-\cos (v) \sin (\mu), \sin (v) \sin (\mu), \cos (\mu)) \\
& \mathbf{S}_{2}=(0, \cos (\kappa), \sin (\kappa)) .
\end{aligned}
$$

The parameters $\nu, \mu$, and $\kappa$ are functions of the exchange parameters and must be determined by minimizing the energy. The E-noncoplanar ${ }_{6}$ configurations have nonzero scalar chirality on the triangle:

$$
\begin{aligned}
\chi= & \left(\mathbf{S}_{0} \times \mathbf{S}_{1}\right) \cdot \mathbf{S}_{2} \\
= & \pm 2 \cos (\nu) \sin (\mu)(-\cos (\kappa) \cos (\mu) \\
& +\sin (\kappa) \sin (\mu) \sin (\nu)) .
\end{aligned}
$$

The magnetization of the configuration lies within the mirror plane which is unbroken when combined with time reversal.

Degenerate spin configurations can be obtained by applying time reversal and lattice symmetries to Eq. (51) and there is a total degeneracy of six. Minimizing the ground state energy with respect to $v, \mu, \kappa$ gives three constraints relating the canting angles to the coupling parameters

$$
\begin{aligned}
\frac{\partial E}{\partial v}= & \Rightarrow \frac{\sin (\mu)}{2}(\cos (\kappa)(3 \cos (v)+\sqrt{3} \sin (v))+2 \sin (\mu) \sin (2 v)) J_{x} \\
+ & \frac{\sin (\mu)}{2}(\cos (\kappa)(\cos (v)-\sqrt{3} \sin (v))+2 \sin (\mu) \sin (2 v)) J_{y} \\
+ & \sin (\mu)(\sqrt{3} \cos (v) \sin (\kappa)+(2 \cos (\mu)+\sin (\kappa)) \sin (v)) D_{y}+2 \sin (\mu)(\cos (2 v) \sin (\mu)+\cos (\kappa) \sin (v)) D_{z} \\
+ & \sin (\mu)(2 \cos (\mu) \cos (v)+\sin (\kappa)(-\cos (v)+\sqrt{3} \sin (v))) K=0 \\
\frac{\partial E}{\partial \mu}= & \Rightarrow \frac{-\cos (\mu)}{2}\left(4 \cos (v)^{2} \sin (\mu)+\cos (\kappa)(\sqrt{3} \cos (v)-3 \sin (v))\right) J_{x} \\
& +\frac{\cos (\mu)}{2}\left(4 \sin (v)^{2} \sin (\mu)+\cos (\kappa)(\sqrt{3} \cos (v)+\sin (v))\right) J_{y}-2(\cos (\mu)+\sin (\kappa)) \sin (\mu) J_{z} \\
& -(\cos (v)(2 \cos (2 \mu)+\cos (\mu) \sin (\kappa))-\sqrt{3}(\cos (\kappa) \sin (\mu)+\cos (\mu) \sin (\kappa) \sin (v))) D_{y} \\
& -(2 \cos (\kappa) \cos (\mu) \cos (v)-\sin (2 \mu) \sin (2 v)) D_{z}+(\cos (\kappa) \sin (\mu) \\
& +2 \cos (2 \mu) \sin (v)-\cos (\mu) \sin (\kappa)(\sqrt{3} \cos (v)+\sin (v))) K=0 \\
\frac{\partial E}{\partial \kappa}= & \Rightarrow \frac{1}{2} \sin (\kappa) \sin (\mu)(\sqrt{3} \cos (v)-3 \sin (\nu)) J_{x}-\frac{1}{2} \sin (\kappa) \sin (\mu)(\sqrt{3} \cos (v)+\sin (v)) J_{y} \\
& +2 \cos (\kappa) \cos (\mu) J_{z}+(\sqrt{3} \cos (\mu) \sin (\kappa)+\cos (\kappa) \sin (\mu)(\sqrt{3} \sin (v)-\cos (v))) D_{y} \\
& +2 \cos (v) \sin (\kappa) \sin (\mu) D_{z}+(\cos (\mu) \sin (\kappa)-\cos (\kappa) \sin (\mu)(\sqrt{3} \cos (v)+\sin (v))) K=0
\end{aligned}
$$

If $v, \mu$, and $\kappa$ are known for a system in the E-noncoplanar ${ }_{6}$ phase, Eqs. (53)-(55) give three constraints on the possible coupling parameters, within the classical description.

Different members of the set of six ground states cannot be placed on neighboring triangles without causing an inconsistency, unless the angles $\mu, v, \kappa$ are fine tuned to special values, via fine tuning of exchange parameters. This confirms that only $\mathbf{q}=0$ configurations are possible within this phase, for generic parameter sets.

\section{E-noncoplanar 12}

Finally, there is the possibility that time reversal, all point group symmetries and all combinations of the two are broken in the ground state, leaving only translation and inversion symmetries intact. In this case the angles $\psi_{\mathrm{E} \alpha}$ [Eqs. (39)-(41)] can take arbitrary values, and symmetry does not fix any relationship between them

$$
\psi_{\mathrm{Ea}} \neq \psi_{\mathrm{Eb}} \neq \psi_{\mathrm{Ec}} .
$$


Moreover $m_{\mathrm{A}_{1}}, m_{\mathrm{A}_{2} \mathrm{a}}, m_{\mathrm{A}_{2} \mathrm{~b}}$ may all be present by symmetry

$$
m_{\mathrm{A}_{1}} \neq 0, \quad m_{\mathrm{A}_{2} \mathrm{a}} \neq 0, \quad m_{\mathrm{A}_{2} \mathrm{~b}} \neq 0 .
$$

The spin directions of the three sites on the triangle have no fixed relationship enforced by symmetry, so there are six parameters in the ground state that can only be determined energetically:

$$
\begin{aligned}
& \mathbf{S}_{0}=\left(\cos \left(\zeta_{0}\right) \sin \left(v_{0}\right), \sin \left(\zeta_{0}\right) \sin \left(v_{0}\right), \cos \left(v_{0}\right)\right) \\
& \mathbf{S}_{1}=\left(\cos \left(\zeta_{1}\right) \sin \left(v_{1}\right), \sin \left(\zeta_{1}\right) \sin \left(v_{1}\right), \cos \left(v_{1}\right)\right) \\
& \mathbf{S}_{2}=\left(\cos \left(\zeta_{2}\right) \sin \left(v_{2}\right), \sin \left(\zeta_{2}\right) \sin \left(v_{2}\right), \cos \left(v_{2}\right)\right) .
\end{aligned}
$$

An example configuration is shown in Fig. 6. The state will generally have nonzero chirality and magnetization in an arbitrary direction. Degenerate spin configurations can be obtained by applying time reversal and lattice symmetries to Eq. (58), giving a total degeneracy of twelve- the maximum possible for a state with translation and inversion symmetries.

As shall be shown using numerics in Sec. IV, this low symmetry state does appear on the ground state phase diagram but only in a very small region of parameter space. Minimizing the energy with respect to $\zeta_{i}, v_{i}(i=0,1,2)$ gives a total of six equations relating the canting angles to the coupling parameters.

$$
\begin{aligned}
\frac{d E}{d \zeta_{i}} & =0 \\
& \Rightarrow \sum_{j \neq i}\left(\begin{array}{c}
-\sin \left(\zeta_{i}\right) \sin \left(v_{i}\right) \\
\cos \left(\zeta_{i}\right) \sin \left(v_{i}\right) \\
0
\end{array}\right) \cdot \mathbf{J}_{i j} \cdot\left(\begin{array}{c}
\cos \left(\zeta_{j}\right) \sin \left(v_{j}\right) \\
\sin \left(\zeta_{j}\right) \sin \left(v_{j}\right) \\
\cos \left(v_{j}\right)
\end{array}\right)=0
\end{aligned}
$$

$$
\begin{aligned}
\frac{d E}{d v_{i}} & =0 \\
& \Rightarrow \sum_{j \neq i}\left(\begin{array}{c}
\cos \left(\zeta_{i}\right) \cos \left(v_{i}\right) \\
\sin \left(\zeta_{i}\right) \cos \left(v_{i}\right) \\
-\sin \left(v_{i}\right)
\end{array}\right) \cdot \mathbf{J}_{i j} \cdot\left(\begin{array}{c}
\cos \left(\zeta_{j}\right) \sin \left(v_{j}\right) \\
\sin \left(\zeta_{j}\right) \sin \left(v_{j}\right) \\
\cos \left(v_{j}\right)
\end{array}\right)=0
\end{aligned}
$$

Thus, if for a system in the E-noncoplanar ${ }_{12}$ phase all six angles are known, it should be possible to use Eqs. (59) and (60) to uniquely determine the six exchange parameters.

\section{PHASE DIAGRAM}

In this section we calculate the ground state phase diagram of Eq. (1) numerically, by comparing optimized energies for the five phases described in Sec. III. The numerical optimization of the energy was done by a combination of random search, simulated annealing, and iterative minimization [40]. Details of the numerics are given in the Appendix.

Figures 7-10 show slices of the phase diagram as a function of $J_{x} /\left|J_{z}\right|$ and $J_{y} /\left|J_{z}\right|$ with $K /\left|J_{z}\right|=\{-0.5,0.5\}$ for both positive (Figs. 7 and 8) and negative (Figs. 9 and 10) $J_{z}$. Each panel in a given figure corresponds to different values of DM interactions $D_{y} /\left|J_{z}\right|$ and $D_{z} /\left|J_{z}\right| . D_{y} /\left|J_{z}\right|$ increases from left to right within each figure and $D_{z} /\left|J_{z}\right|$ from bottom to top. Taken together, Figs. 7-10 give a broad view of the competition between different magnetic orders as anisotropic exchange parameters are varied. Further phase diagrams for a greater range and variety of parameter sets are shown in the Supplemental Material [41].

The boundaries of the $A_{1}$ and $A_{2}$ phases can also be calculated analytically using conditions (27) and (28). These analytic boundaries are shown as white lines in Figs. 7-10 and agree with the results of the numerics. The boundaries between the different $E$ phases are only calculated numerically.

One notable feature of Figs. 7-10 is that the E-coplanar phase is generally found bordering the $A_{1}$ phase, whereas the E-noncoplanar ${ }_{6}$ phase is generally found bordering the $A_{2}$ phase. This is natural since the E-coplanar phase mixes in a finite value of the $A_{1}$ order parameter and likewise the $E$-noncoplanar ${ }_{6}$ includes a finite $A_{2}$ order parameter.

Another striking feature of the phase diagram is the rarity of the E-noncoplanar ${ }_{12}$ phase. This low-symmetry configuration occupies only small portions of the phase diagrams in Figs. 7-10, with its stability generally being increased by a strong negative value of $D_{z}$.

To investigate the relative frequency of the different phases in the overall parameter space we have calculated the ground state for 100000 different parameter sets, randomly chosen from a uniform distribution on the surface of the sixdimensional hypersphere defined by

$$
J_{x}^{2}+J_{y}^{2}+J_{z}^{2}+D_{y}^{2}+D_{z}^{2}+K^{2}=1 .
$$

The pie chart in Fig. 11(a) shows the relative frequency of each of the five phases obtained from this procedure. It confirms that E-noncoplanar ${ }_{12}$ is indeed a rare phase, found as the ground state for only $\sim 0.5 \%$ of randomly generated parameter sets. The four other phases are comparatively common.

This leads us to conclude although the E-noncoplanar ${ }_{12}$ state does not require perfect fine tuning to be realized in a kagome material (i.e., it occupies a finite fraction of parameter space), it is unlikely to be realized serendipitously. The other four phases should constitute the classical ground states for the vast majority of kagome materials to which the theory in this paper can be applied (i.e., those with nearest-neighbor, anisotropic interactions).

The above assumes a probability distribution of parameter sets which is isotropic in the six-dimensional space $\left(J_{x}, J_{y}, J_{z}, D_{y}, D_{z}, K\right)$. This may not be the case physically, and indeed it is frequently assumed that the off-diagonal components of the exchange tensor $D_{y}, D_{z}, K$ should be smaller than the diagonal ones $J_{x}, J_{y}, J_{z}$. We have investigated the distribution of ground states under this assumption, by generating 100000 random parameter sets by choosing $J_{x}, J_{y}, J_{z}$ from a uniform distribution on the surface of the unit sphere:

$$
J_{x}^{2}+J_{y}^{2}+J_{z}^{2}=1
$$

and independently choosing $D_{y}, D_{z}, K$ from a uniform distribution on the surface of a smaller sphere:

$$
D_{y}^{2}+D_{z}^{2}+K^{2}=0.1 \text {. }
$$

The resulting distribution of ground states is shown in Fig. 11(b). The relative frequency of different phases is very 


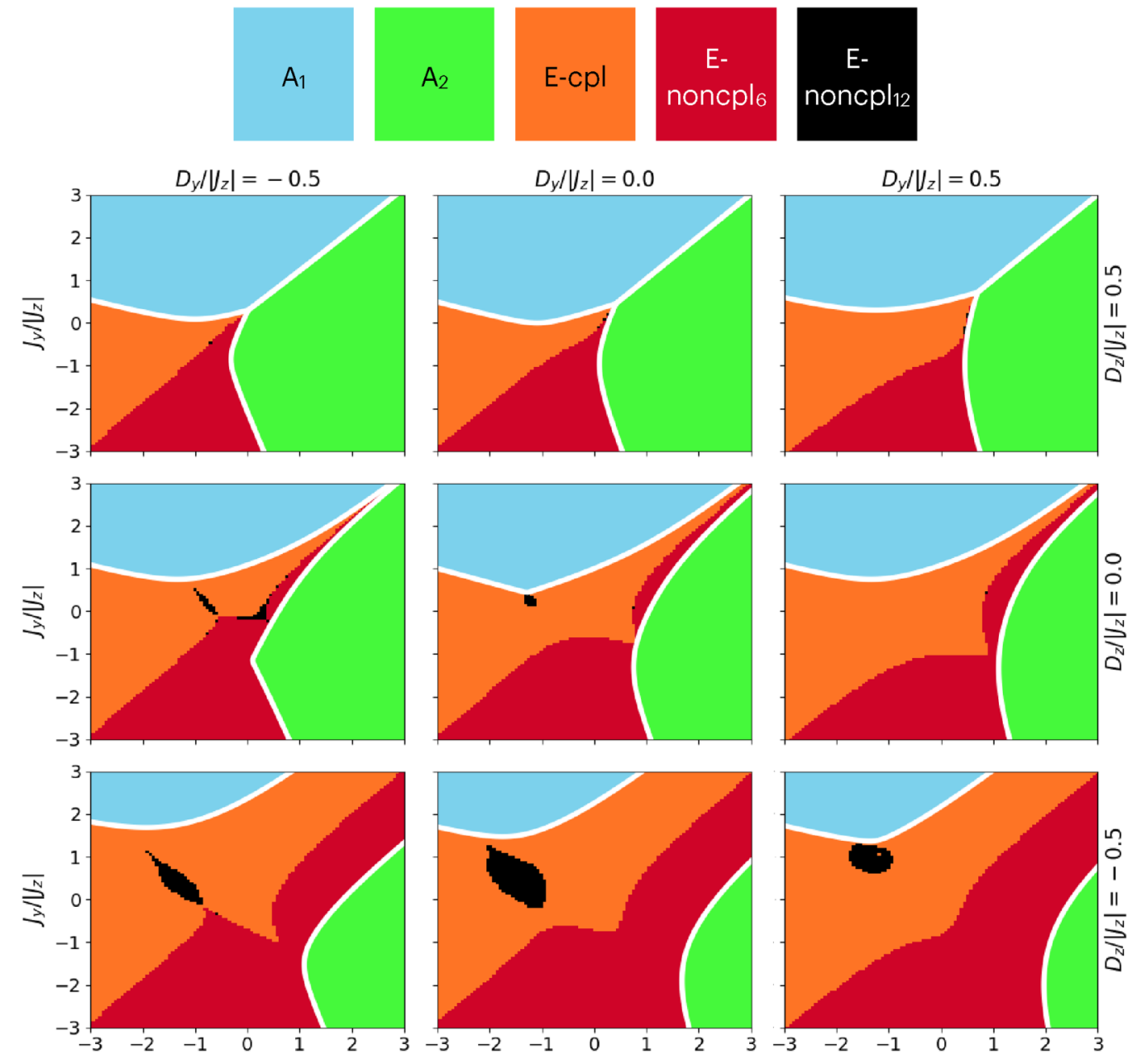

FIG. 7. $T=0$ phase diagram with $J_{z}>0$ and $K=-0.5\left|J_{z}\right|$. Each panel shows a slice of the phase diagram as a function of $J_{x}$ and $J_{y}$ for different, fixed, values of the DM directions $D_{y}$ and $D_{z}$, with $D_{y}$ increasing from left to right and $D_{z}$ from bottom to top. The phase diagram is obtained by comparing numerically optimized energies for the five phases described in Sec. III. The numerical optimization procedure is described in the Appendix. The white lines show analytic calculations of the boundaries of the $A_{1}$ and $A_{2}$ phases, using conditions (27) and (28).

similar to that with an isotropic distribution of parameters, although the prevalence of the E-noncoplanar ${ }_{12}$ phase increases from $\sim 0.5 / \%$ to $\sim 2 / \%$.

\section{A. Phase diagram in the vicinity of the antiferromagnetic Heisenberg limit}

The limit $J_{x}=J_{y}=J_{z}=J>0, D_{y}=K=D_{z}=0$, gives the well studied nearest neighbor antiferromagnetic Heisenberg model, which is known to have a highly degenerate ground state [39]. Generic perturbations away from this limit lift the degeneracy, stabilizing a ground state which is unique up to global symmetry operations.

Figure 12 shows the effect of perturbing the Heisenberg model with finite off-diagonal couplings $D_{y}, D_{z}, K . D_{z}>0$ strongly favors $\mathrm{A}_{2}$ order, while $D_{z}<0$ favors ordering into the E-coplanar or E-noncoplanar ${ }_{6}$ phases depending on which of $D_{y}$ or $K$ is the more dominant perturbation. Our results are in agreement with those of Elhajal et al. [21], who considered the case of perturbing the Heisenberg model with Dzyaloshinskii-Moriya interactions $D_{y}, D_{z}$, fixing $K=0$.

When comparing the results here with those of Ref. [21] one should note that the ground state configurations of the E-noncoplanar ${ }_{6}$ phase become coplanar in the limit of strong positive $J$ and $K=0$. This agrees with the labeling of the same phase as coplanar in Ref. [21]. Once all symmetry allowed couplings (particularly $K$ ) are present, this phase becomes noncoplanar, as identified here.

It is notable that the $A_{1}$ phase does not appear at all in Fig. 12. This can be readily understood from the couplings in Eqs. (14)-(20) when $J_{x}=J_{y}, \lambda_{\mathrm{A}_{1}}=\lambda_{\mathrm{A}_{2} \text {,bb }}$. This then implies that $\omega_{\mathrm{A}_{2} 0} \leqslant \lambda_{\mathrm{A}_{1}}$ [cf. Eqs. (25) and (27)] with the equality only applying when $\lambda_{\mathrm{A}_{2}, \mathrm{ab}}=2\left(\sqrt{3} D_{y}+K\right)=0$.

Thus, when $J_{x}=J_{y}$ the $A_{2}$ phase will quite generally have a lower energy than the $A_{1}$ phase. A necessary (but not sufficient) condition for the $A_{1}$ configurations to be the sole ground states is that $\lambda_{\mathrm{A}_{1}}<\lambda_{\mathrm{A}_{2} \mathrm{bb}} \Rightarrow J_{x}<J_{y}$.

The effect of allowing small anisotropy in the transverse exchanges $J_{x}, J_{y}$ is illustrated in Fig. 13. Here we 


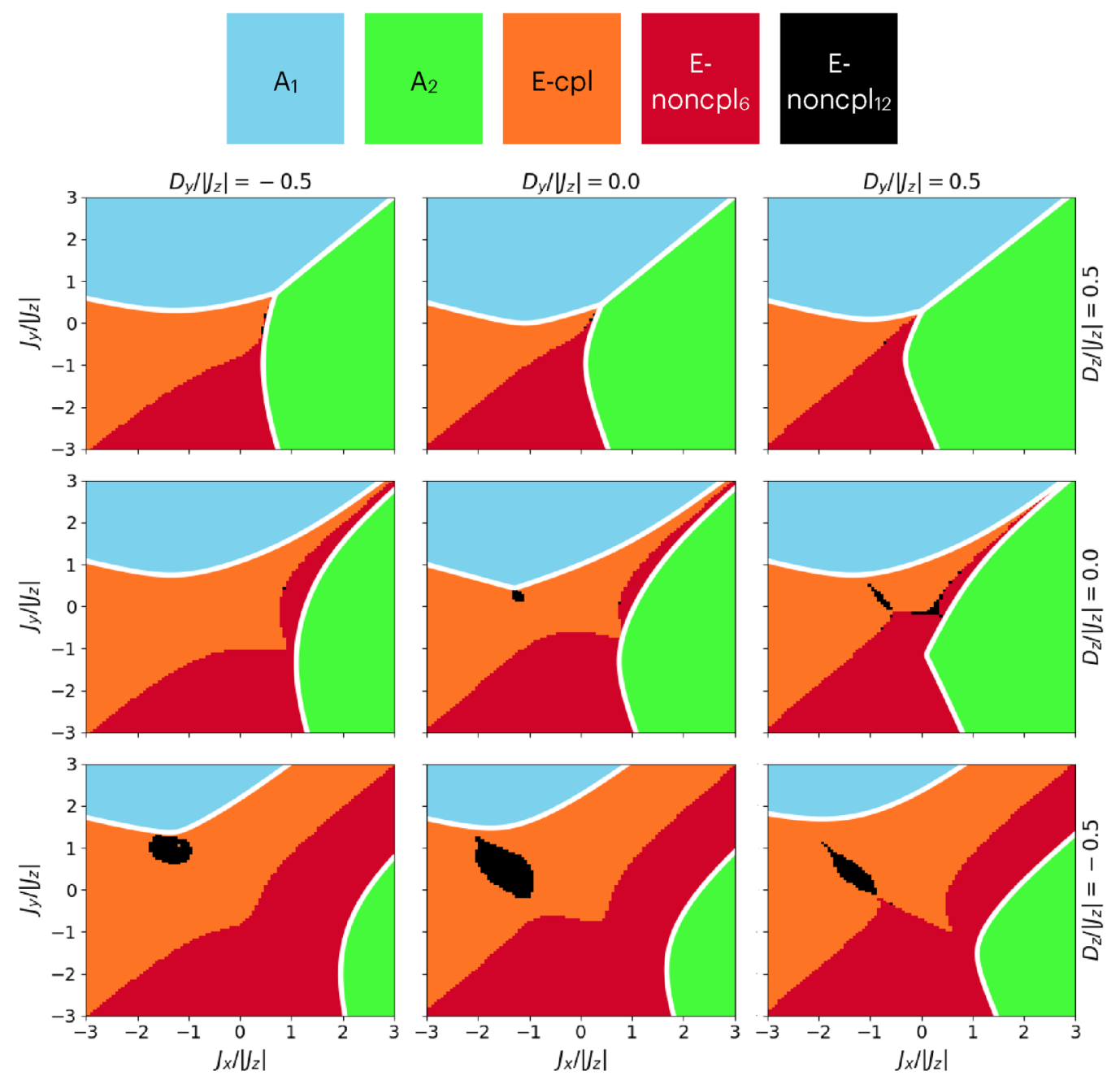

FIG. 8. $T=0$ phase diagram with $J_{z}>0$ and $K=0.5\left|J_{z}\right|$. Each panel shows a slice of the phase diagram as a function of $J_{x}$ and $J_{y}$ for different, fixed, values of the DM directions $D_{y}$ and $D_{z}$, with $D_{y}$ increasing from left to right and $D_{z}$ from bottom to top. The phase diagram is obtained by comparing numerically optimized energies for the five phases described in Sec. III. The numerical optimization procedure is described in the Appendix. The white lines show analytic calculations of the boundaries of the $A_{1}$ and $A_{2}$ phases, using conditions (27) and (28).

set

$$
D_{z}=0, J_{z}=J>0, J_{x}=J+\frac{\delta J_{\perp}}{2}, J_{y}=J-\frac{\delta J_{\perp}}{2}
$$

and vary $D_{y} / J$ and $K / J$. As implied by the discussion above, $\delta J_{\perp}<0$ favors $A_{1}$ order, becoming unstable to the $\mathrm{E}$ coplanar phase on increasing $K$. Conversely, $\delta J_{\perp}>0$ favors $\mathrm{A}_{2}$ order, which gives way to the E-noncoplanar ${ }_{6}$ phase for strong $K$.

\section{RELEVANCE TO KAGOME MATERIALS}

In this section we discuss the application of our results to real kagome materials. We divide our discussion into two areas: Firstly, rare-earth magnets belonging to the family $\mathrm{R}_{3} \mathrm{~A}_{2} \mathrm{Sb}_{3} \mathrm{O}_{14}$ [13-20] (sometimes referred to as "tripod kagome" materials [15,17]), and secondly $\mathrm{Cu}, \mathrm{Fe}$, and $\mathrm{Cr}$ based magnets where exchange anisotropy should be weaker but nevertheless plays a role in ground state selection. Aside from the systems mentioned below, we anticipate that ongoing work in synthesizing frustrated magnets with strong spin-orbit coupling will reveal new kagome systems to which our results can be applied in the coming years.

\section{A. $\mathrm{R}_{3} \mathrm{~A}_{2} \mathrm{Sb}_{3} \mathrm{O}_{14}$ family}

In the last few years several rare-earth kagome materials with the general formula $\mathrm{R}_{3} \mathrm{~A}_{2} \mathrm{Sb}_{3} \mathrm{O}_{14}$ have been synthesized. This includes materials with $\mathrm{A}=\mathrm{Mg}, \mathrm{Zn}$ and $\mathrm{R}=\mathrm{Pr}, \mathrm{Nd}, \mathrm{Sm}$, Eu, Gd, Tb, Dy, Ho, Er, Tm, Yb [13,14,17,19].

Where $\mathrm{R}$ is a non-Kramers ion (Pr, Eu, Tb, Ho, Tm), the crystal electric field (CEF) will generally have a nonmagnetic singlet ground state, due to the low symmetry of the rare-earth environment. If the gap between this singlet and higher CEF states is smaller than or comparable to the energy scale of interactions, interesting physics may ensue. If the CEF gap is large, the overall ground state of the system will be a trivial singlet driven by the onsite physics. Either way, Eq. (1) cannot describe such physics without being augmented by additional 


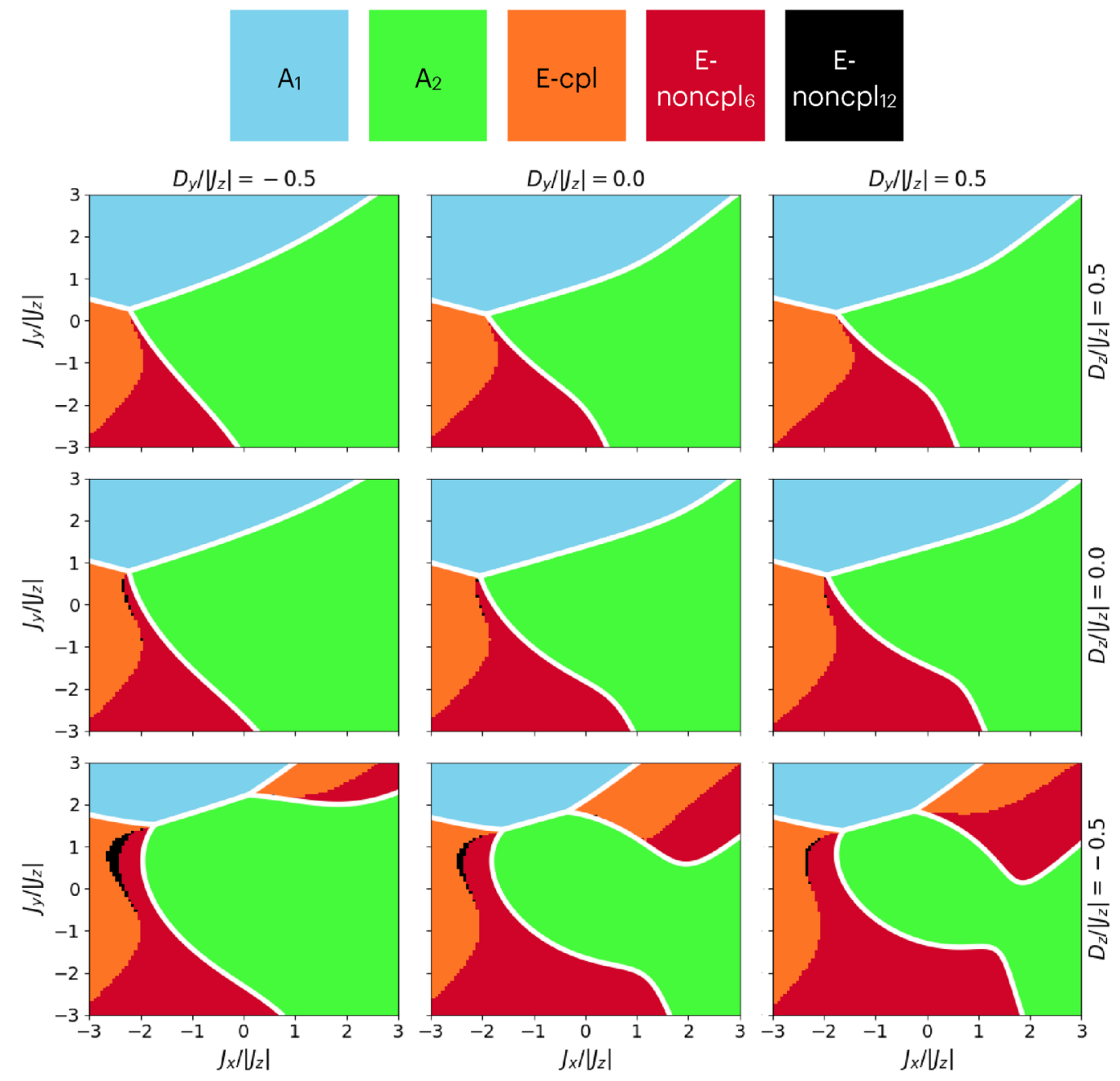

FIG. 9. $T=0$ phase diagram with $J_{z}<0$ and $K=-0.5\left|J_{z}\right|$. Each panel shows a slice of the phase diagram as a function of $J_{x}$ and $J_{y}$ for different, fixed, values of the DM directions $D_{y}$ and $D_{z}$, with $D_{y}$ increasing from left to right and $D_{z}$ from bottom to top. The phase diagram is obtained by comparing numerically optimized energies for the five phases described in Sec. III. The numerical optimization procedure is described in the Appendix. The white lines show analytic calculations of the boundaries of the $A_{1}$ and $A_{2}$ phases, using conditions (27) and (28).

terms, so we will not discuss non-Kramers materials further here.

Where $R$ is a Kramers ion, the CEF will split the $2 J+1$ multiplet into a series of doublets. At energy and temperature scales below the gap between the lowest and first excited doublet, the magnetism may be represented by pseudospin-1/2 operators $\mathbf{S}_{i}$. $\mathbf{S}_{i}$ does not correspond precisely to the magnetic moment, but relates to it via the $g$ tensor [Eq. (2)]. The important thing for our purposes is that $\mathbf{S}_{i}$ transforms like a magnetic moment with respect to timereversal and lattice symmetries, in which case Eqs. (1)-(6) describe the exchange interactions. Below we briefly discuss the various members of the $\mathrm{R}_{3} \mathrm{~A}_{2} \mathrm{Sb}_{3} \mathrm{O}_{14}$ family, with Kramers ions $R$, in the light of the predictions made in this paper.

The scalar chiral order observed in $\mathrm{Nd}_{3} \mathrm{Mg}_{2} \mathrm{Sb}_{3} \mathrm{O}_{14}$ [16,20] corresponds precisely to the $\mathrm{A}_{2}$ phase predicted in this work. The magnetic order of the sister compound $\mathrm{Nd}_{3} \mathrm{Zn}_{2} \mathrm{Sb}_{3} \mathrm{O}_{14}$ has not yet been characterized, but given its essentially similar thermodynamic properties [17] and crystal field en- vironment [18] it seems likely to fall in the same phase as $\mathrm{Nd}_{3} \mathrm{Mg}_{2} \mathrm{Sb}_{3} \mathrm{O}_{14}$.

$\mathrm{Er}_{3} \mathrm{Mg}_{2} \mathrm{Sb}_{3} \mathrm{O}_{14}$ was reported in Ref. [17] to avoid long range order down to very low temperatures. It thus appears to be a candidate spin liquid material. The regions near the phase boundaries of the classical phase diagram presented here are likely to be particularly fertile ground for the formation of spin liquid states, and this will be an interesting direction for future research. $\mathrm{Er}_{3} \mathrm{Zn}_{2} \mathrm{Sb}_{3} \mathrm{O}_{14}$ exhibits strong structural disorder and associated glassy behavior of the magnetic properties [17], which is beyond the scope of our present discussion.

$\mathrm{Yb}_{3} \mathrm{Mg}_{2} \mathrm{Sb}_{3} \mathrm{O}_{14}$ exhibits long range order at $T_{N} \approx 0.88 \mathrm{~K}$ [17]. The form of this magnetic order has yet to be reported in the literature. Based on the expectation that, as a rare earth magnet with moderate magnetic moment, the theory in this paper should be applicable to $\mathrm{Yb}_{3} \mathrm{Mg}_{2} \mathrm{Sb}_{3} \mathrm{O}_{14}$, we expect that the order will be one of the states discussed in this work. Like $\mathrm{Er}_{3} \mathrm{Zn}_{2} \mathrm{Sb}_{3} \mathrm{O}_{14}, \mathrm{Yb}_{3} \mathrm{Zn}_{2} \mathrm{Sb}_{3} \mathrm{O}_{14}$ has strong structural disorder, although unlike the Er compound it does not show clear signs of spin freezing [17]. 


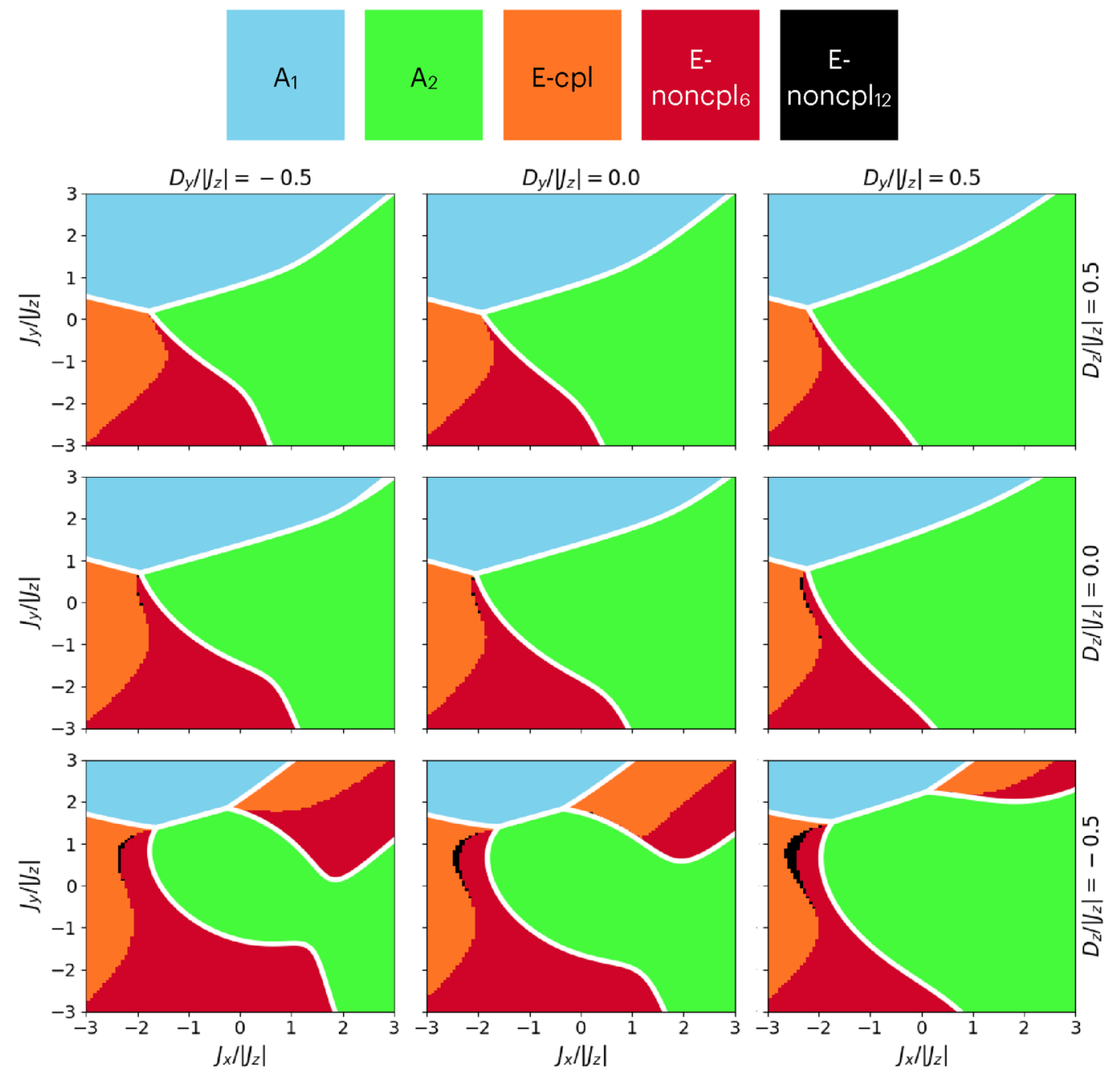

FIG. 10. $T=0$ phase diagram with $J_{z}<0$ and $K=0.5\left|J_{z}\right|$. Each panel shows a slice of the phase diagram as a function of $J_{x}$ and $J_{y}$ for different, fixed, values of the DM directions $D_{y}$ and $D_{z}$, with $D_{y}$ increasing from left to right and $D_{z}$ from bottom to top. The phase diagram is obtained by comparing numerically optimized energies for the five phases described in Sec. III. The numerical optimization procedure is described in the Appendix. The white lines show analytic calculations of the boundaries of the $A_{1}$ and $A_{2}$ phases, using conditions (27) and (28).

$\mathrm{Sm}_{3} \mathrm{Mg}_{2} \mathrm{Sb}_{3} \mathrm{O}_{14}$ [13] and $\mathrm{Sm}_{3} \mathrm{Zn}_{2} \mathrm{Sb}_{3} \mathrm{O}_{14}$ [14] have both been synthesized but their low temperature magnetism has yet to be characterized in detail. This may be challenging due to the small magnetic moment of the $\mathrm{Sm}^{3+}$ ion, but recent experiments on the pyrochlores $\mathrm{Sm}_{2} \mathrm{Ti}_{2} \mathrm{O}_{7}$ and $\mathrm{Sm}_{2} \mathrm{Sn}_{2} \mathrm{O}_{7}$ indicate that this is possible [42]. There is some evidence of hysteresis in the low temperature magnetization curve for $\mathrm{Sm}_{3} \mathrm{Zn}_{2} \mathrm{Sb}_{3} \mathrm{O}_{14}$ [14] but not for $\mathrm{Sm}_{3} \mathrm{Mg}_{2} \mathrm{Sb}_{3} \mathrm{O}_{14}$ [13], which may provide some clue as to the low temperature state.

Materials with $\mathrm{R}=\mathrm{Gd}$ present a somewhat different case, because Hund's rules imply vanishing orbital angular momentum $L=0$ for the $\mathrm{Gd}^{3+}$ ion. The magnetism on the $\mathrm{Gd}$ sites thus comes from a pure $S=7 / 2$ spin and anisotropies in the interactions should be much weaker. Some understanding of this case can be gained from considering a model with nearest neighbor Heisenberg exchange and the nearest-neighbor part of the dipolar interaction:

$$
\mathcal{H}=J \sum_{\langle i j\rangle} \mathbf{S}_{i} \cdot \mathbf{S}_{j}+\tilde{D}_{\mathrm{nn}} \sum_{\langle i j\rangle}\left(\mathbf{S}_{i} \cdot \mathbf{S}_{j}-3 \mathbf{S}_{i} \cdot \hat{\mathbf{r}}_{i j} \mathbf{S}_{j} \cdot \hat{\mathbf{r}}_{i j}\right) .
$$

In terms of the symmetry-allowed interaction matrices [Eqs. (4)-(6)] this Hamiltonian corresponds to setting

$$
\begin{aligned}
& J_{x}=J-2 \tilde{D}_{\mathrm{nn}}, J_{y}=J_{z}=J+\tilde{D}_{\mathrm{nn}}, \\
& D_{y}=D_{z}=K=0 .
\end{aligned}
$$

Inserting Eq. (65) into Eqs. (14)-(23) leads us to the conclusion that for $J, \tilde{D}_{\mathrm{nn}}>0$, the $A_{1}$ configuration is favored out of the forms of order considered in this paper. This agrees with the conclusions of Maksymenko et al. [43], who studied the phase diagram incorporating isotropic nearest neighbor exchange $J$ with the full long ranged dipolar interaction $D$ and found the $A_{1}$ configuration as the ground state for weak to moderate $D$ and antiferromagnetic $J$. It also agrees with previous predictions about the ground state of $\mathrm{Gd}_{3} \mathrm{Mg}_{2} \mathrm{Sb}_{3} \mathrm{O}_{14}$ [15] and with the observed antiferromagnetic transition at $T_{N} \approx 1.7 \mathrm{~K}[15,44]$, although differences between the field cooled and zero-field cooled susceptibility [44] remain to be understood.

For $\mathrm{R}=\mathrm{Dy}$ the ionic magnetic moment is very large and the long range component of the dipolar interaction cannot 


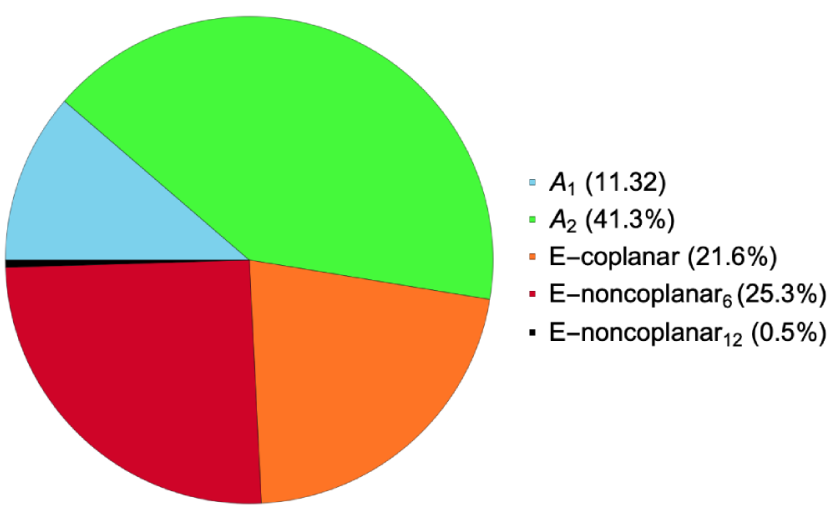

(a)

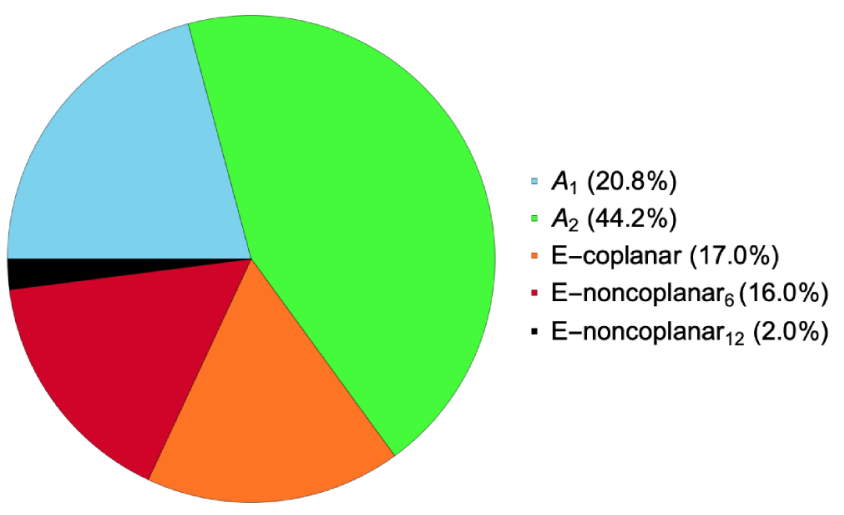

(b)

FIG. 11. Relative frequency of different phases within the full parameter space of the Hamiltonian [Eq. (1)], with exchange parameters generated randomly from two different distributions. (a) Exchange parameters are generated randomly according to a uniform distribution on the surface of the six-dimensional hypersphere defined by Eq. (61). (b) Diagonal exchange parameters $J_{x}, J_{y}, J_{z}$ are generated according to a uniform distribution on the surface of a sphere with unit radius, and off-diagonal $D_{y}, D_{z}, K$ exchange parameters are generated independently from a uniform distribution on the surface of a sphere with radius $=0.1$ [Eqs. (62) and (63)]. This models the effect of the assumption that the scale of off-diagonal couplings is lower. The effect on the distribution of phases is minor overall, although assuming weaker off-diagonal exchange expands the size of the rare E-noncoplanar ${ }_{12}$ from $\sim 0.5 \%$ to $\sim 2 \%$. In each case, frequencies are determined by numerically finding the ground state for 100000 random parameter sets generated according to the stated distributions.

be ignored. $\mathrm{Dy}_{3} \mathrm{Mg}_{2} \mathrm{Sb}_{3} \mathrm{O}_{14}$ exhibits an unusual "fragmented" [45] phase where there is an ordering of emergent "charge" degrees of freedom while spins remain partially disordered [46]. The long-range dipole-dipole interaction plays a crucial role in this phenomenon $[47,48]$ and thus it is beyond the scope of the theory presented in this paper.

\section{B. Nearly isotropic systems}

While the most obvious application of the results in this paper is found in systems where exchange anisotropy is strong, our results can also be applied to understand cases where isotropic Heisenberg exchange is weakly perturbed by short ranged anisotropic interactions. This is the case in the $\mathrm{Fe}$ and $\mathrm{Cr}$ jarosites $\mathrm{AM}_{3}(\mathrm{OH})_{6}\left(\mathrm{SO}_{4}\right)_{2}$ where $\mathrm{M}=\{\mathrm{Fe}, \mathrm{Cr}\}$ and $\mathrm{A}=\left\{\mathrm{K}, \mathrm{Rb}, \mathrm{NH}_{4}, \mathrm{Na}\right\}$ [31-35]. These are found to order in the $A_{2}$ phase-the most prevalent of our phase diagram. This is generally understood to be a consequence of antiferromagnetic Heisenberg exchange perturbed by a weak $D_{y}$. This interpretation fully agrees with the results presented here: It can readily be checked that inserting

$$
\begin{aligned}
& J_{x}=J_{y}=J_{z}=J>0 \\
& D_{z}=K=0, \quad\left|D_{y}\right| \ll J
\end{aligned}
$$

into Eqs. (14)-(23) gives an outcome obeying condition (28) and hence a ground state in the $A_{2}$ phase [cf. Fig. 12]. What this work adds to the discussion is a simple and systematic approach to finding the preferred ground state for general kinds of anisotropic nearest neighbor perturbation.

An example where weak anisotropic perturbations away from a Heisenberg model lead to something other than $A_{2}$ order is given by $\mathrm{Cd}$ kapellasite [36]. The weak ferromagnetic moment confined within the kagome planes in that material is only consistent with the E-coplanar phase, out of the phases in this paper.

\section{SUMMARY AND DISCUSSION}

In this paper we have developed a theory of the magnetic orders induced by nearest-neighbor exchange anisotropy in kagome magnets. Our theory reveals that five distinct magnetic orders can be expected from such interactions, all retaining the translational symmetry of the lattice, but being distinguished from one another by their transformations under time-reversal and point group symmetries. The five phases are: $A_{1}$ (Fig. 2), $A_{2}$ (Fig. 3), E-coplanar (Fig. 4), E-noncoplanar 6 (Fig. 5), E-noncoplanar ${ }_{12}$ (Fig. 6). They are labeled according to the irreducible representation of the point group $C_{3 v}$ with which the primary order parameter transforms, their coplanar or noncoplanar nature and their degeneracy. Equations (27) and (28) give exact conditions for the $A_{1}$ and $A_{2}$ configurations to be classical ground states.

We have used numerical calculations to determine the full zero temperature phase diagram of the most general anisotropic nearest-neighbor exchange model, showing the extent of these five phases (Figs. 7-10). One of the five phases $\left(E\right.$-noncoplanar $\left.{ }_{12}\right)$ is found to be exceedingly rare in the parameter space (Fig. 11).

We have discussed how this theory relates to various real kagome materials (Sec. V), with both strong and weak exchange anisotropy. The dominance of noncollinear $\left(A_{1}\right.$, E-coplanar) and noncoplanar $\left(A_{2}\right.$, E-noncoplanar $\left.{ }_{6,12}\right)$ states on the phase diagram suggests a high possibility of spin excitations with topological band structures in many kagome materials [49-51]. It is likely that the five phases identified here from analysis of broken symmetries can be subdivided further by the topology of the excitation bands. The possibility of coupling to itinerant electrons is an interesting area for future research with a view to investigating topological transport phenomena. 


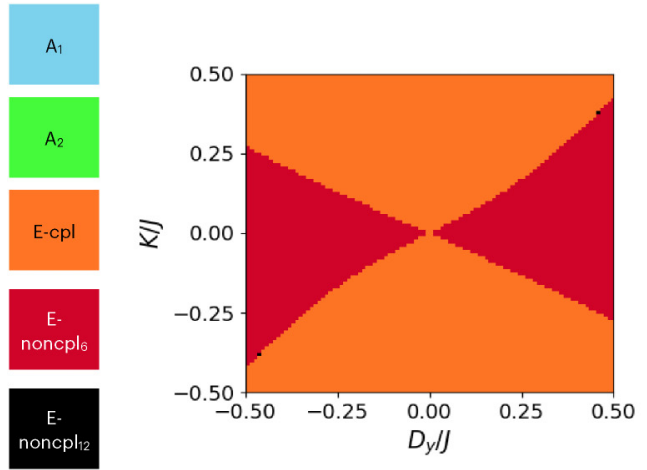

(a) $D_{z}=-0.25 \mathrm{~J}$



(b) $D_{z}=0$

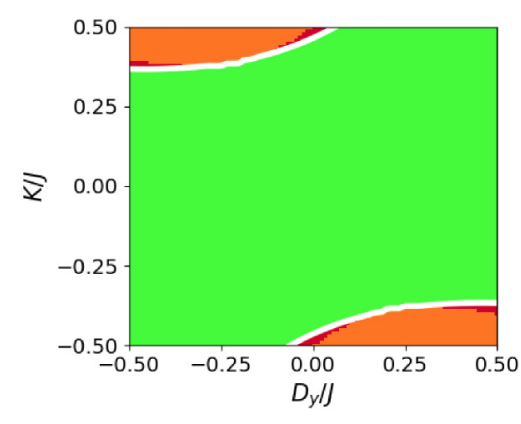

(c) $D_{z}=0.25 \mathrm{~J}$

FIG. 12. Ground state phase diagram obtained from perturbing the antiferromagnetic Heisenberg model $\left(J_{x}=J_{y}=J_{z}=J>0\right)$ with offdiagonal couplings $D_{y}, D_{z}, K$. Phase diagrams are shown as a function of $D_{y} / J, K / J$ at fixed values of $D_{z} / J=-0.25$ (a), $D_{z} / J=0$ (b), $D_{z} / J=0.25$ (c). The phase diagram is obtained by comparing numerically optimized energies for the five phases described in Sec. III. The numerical optimization procedure is described in the Appendix. The $A_{1}$ phase does not appear on these phase diagrams, as it can only be stabilized as a unique ground state when $J_{x}<J_{y}$, whereas $J_{x}=J_{y}$ here. The white lines show analytic calculations of the boundaries of the $\mathrm{A}_{2}$ phase, using condition (28).

The approach used in this work relies on the ability to decompose the Hamiltonian into a sum over blocks, such that the ground state is obtained by finding the ground state on each block and tiling it over the lattice. This would seem to limit the usefulness of the approach for systems with further neighbor interactions, since such a decomposition may either not be possible or may require such large blocks that the decomposition is no longer a useful simplification. Applying the method from this work to quantum systems will also not be possible in general - even for nearest neighbor interactionsbecause the Hamiltonians on neighboring blocks will usually not commute. There are, however, some specific, fine-tuned, cases where the exact ground state of a quantum system can be built up by such a block-by-block approach [28,52].

While we have restricted ourselves here to phases which are stable over finite regions of the classical phase diagram, a study of the phase boundaries may also be interesting. As has been studied elsewhere [7,8] phase boundaries between competing classical phases can host nontrivial enlarged manifolds of zero-energy states, which in some cases are associated with new forms of spin liquid [53]. In general, the greater the degree of degeneracy around the phase boundary, the more favorable the situation becomes towards the formation of spin liquids. Different phase boundaries will have different amounts of additional degeneracy and so some will be more favorable for spin liquid formation than others. Boundaries where 3 (rather than just 2) phases meet may host particularly interesting physics as seen in, e.g., Ref. [53]. An analysis of each possible phase boundary would be an interesting undertaking, which we leave open for future work.

\section{ACKNOWLEDGMENTS}

The author thanks Zhiling Dun, Karim Essafi, Ludovic Jaubert, and Han Yan for helpful discussions and collab-





(a) $\delta J_{\perp}=-0.25 J$

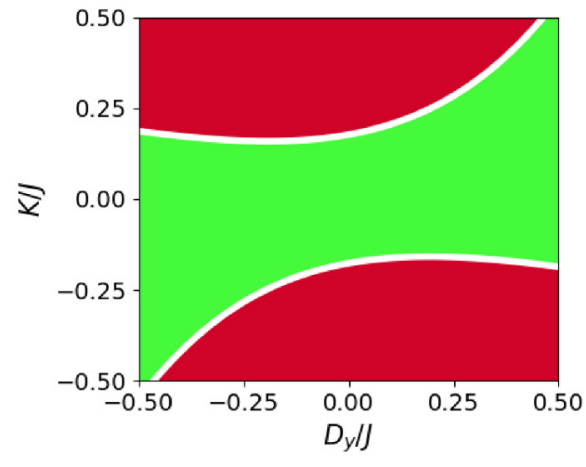

(b) $\delta J_{\perp}=0.25 \mathrm{~J}$

FIG. 13. Ground state phase diagram obtained from perturbing the antiferromagnetic Heisenberg model with off-diagonal couplings $D_{y}, K$, and anisotropy in the transverse exchange $J_{x}=J+\frac{\delta J_{\perp}}{2}, J_{y}=J-\frac{\delta J_{\perp}}{2}$. We set $J_{z}=J>0$ and $D_{z}=0$ in both panels. Phase diagrams are shown as a function of $D_{y} / J, K / J$ at fixed values of $\delta J_{\perp} / J=-0.25$ (a), $\delta J_{\perp} / J=0.25$ (b). The phase diagram is obtained by comparing numerically optimized energies for the five phases described in Sec. III. The numerical optimization procedure is described in the Appendix. The white lines show analytic calculations of the boundaries of the $A_{1}$ and $A_{2}$ phases, using conditions (27) and (28). 
orations on related work. Karim Essafi, Ludovic Jaubert, and Johannes Richter are also thanked for feedback on the draft manuscript. The author acknowledges the hospitality of LOMA at the University of Bordeaux, where part of this work was carried out.

\section{APPENDIX: NUMERICAL OPTIMIZATION OF ENERGIES}

Here we describe the numerical optimization used to obtain the phase diagrams in Figs. 7-10 and the estimates of the relative frequency of phases in Fig. 11. For a given parameter set, the energy is optimized separately for each of the five phases described in Sec. III and then the optimized energies are compared to determine which is the lowest. Due to the argument in Sec. III A, we need only optimize the configuration on a single triangle, since we know that a ground state on the full lattice can be obtained by tiling the ground state of a single triangle everywhere.

The optimization for each phase is done by either random search or simulated annealing combined with iterative minimization [40], apart from the $A_{1}$ phase where the spin configuration is fixed [Eq. (33)] and thus the corresponding energy can directly be calculated without any optimization being necessary:

$$
E_{\mathrm{A}_{1}}=\frac{3}{4}\left(-2 \sqrt{3} D_{z}+J_{x}-3 J_{y}\right) .
$$

For the other four phases $\left(A_{2}, E\right.$-coplanar, $E$-noncoplanar ${ }_{6}$, E-noncoplanar ${ }_{12}$ ), the optimization procedure is as described below.

\section{Optimizing $A_{2}$ configuration}

The form for the $A_{2}$ configurations is given in Eq. (34). This can be written as

$$
\begin{gathered}
\mathbf{S}_{0}=\left(-\frac{\sqrt{3}}{2} s_{a}, s_{a} / 2, s_{b}\right) \\
\mathbf{S}_{1}=\left(\frac{\sqrt{3}}{2} s_{a}, s_{a} / 2, s_{b}\right) \\
\mathbf{S}_{2}=\left(0,-s_{a}, s_{b}\right)
\end{gathered}
$$

with $\left(s_{a}, s_{b}\right)$ on the unit circle

$$
s_{a}^{2}+s_{b}^{2}=1
$$

Initially, we calculate the energy for $10^{5}$ randomly generated values of $\left(s_{a}, s_{b}\right)$ on the unit circle. The lowest energy configuration obtained from this random search is then used as input for the iterative minimization step.

In the iterative minimization step $\left(s_{a}, s_{b}\right)$ are updated as

$$
\begin{gathered}
s_{a} \rightarrow \frac{s_{a}-c \frac{\partial E}{\partial s_{a}}}{\left|\left(s_{a}-c \frac{\partial E}{\partial s_{a}}, s_{b}-c \frac{\partial E}{\partial s_{b}}\right)\right|} \\
s_{b} \rightarrow \frac{s_{b}-c \frac{\partial E}{\partial s_{b}}}{\left|\left(s_{a}-c \frac{\partial E}{\partial s_{a}}, s_{b}-c \frac{\partial E}{\partial s_{b}}\right)\right|} .
\end{gathered}
$$

For sufficiently small, positive $c$ this update is guaranteed to reduce the energy, unless the system is already in a locally optimal configuration before the update.
The parameter $c$ is initially set to 0.1 . If the update (A6) does not reduce the energy then $c$ is reduced by a factor of 2 and the update is attempted again. This procedure is repeated until the configuration converges.

\section{Optimizing E-coplanar configuration}

The form for an E-coplanar configuration is given in Eq. (45). This can be rewritten as

$$
\begin{gathered}
\mathbf{S}_{0}=\left(\sigma_{x}, \sigma_{y}, \sigma_{z}\right) \\
\mathbf{S}_{1}=\left(\sigma_{x},-\sigma_{y},-\sigma_{z}\right) \\
\mathbf{S}_{2}=(1,0,0)
\end{gathered}
$$

with $\left(\sigma_{x}, \sigma_{y}, \sigma_{z}\right)$ on the unit sphere

$$
\sigma_{x}^{2}+\sigma_{y}^{2}+\sigma_{z}^{2}=1
$$

Initially, we calculate the energy for $10^{5}$ randomly generated values of $\left(\sigma_{x}, \sigma_{y}, \sigma_{z}\right)$ on the unit sphere. The lowest energy configuration obtained from this random search is then used as input for the iterative minimization step.

In the iterative minimization step $\left(\sigma_{x}, \sigma_{y}, \sigma_{z}\right)$ are updated as

$$
\sigma_{\alpha} \rightarrow \frac{\sigma_{\alpha}-c \frac{\partial E}{\partial \sigma_{\alpha}}}{\left|\left(\sigma_{x}-c \frac{\partial E}{\partial \sigma_{x}}, \sigma_{y}-c \frac{\partial E}{\partial \sigma_{y}}, \sigma_{z}-c \frac{\partial E}{\partial \sigma_{z}}\right)\right|} .
$$

The parameter $c$ is initially set to 0.1 . If the update (A11) does not reduce the energy then $c$ is reduced by a factor of 2 and the update is attempted again. This procedure is repeated until the configuration converges.

The set of configurations covered by the E-coplanar ansatz (45) includes the $A_{1}$ configurations (when $\phi=\frac{4 \pi}{3}, \theta=\frac{\pi}{2}$ ). Because of this, if the $E$-coplanar optimization is found to give the lowest energy of the five possibilities we must check that the obtained configuration has a nonzero value of at least one of the order parameters $\mathbf{m}_{\mathrm{E} \alpha}$. In practice we check that

$$
\left|\mathbf{m}_{\mathrm{E} a}\right|^{2}+\left|\mathbf{m}_{\mathrm{E} b}\right|^{2}+\left|\mathbf{m}_{\mathrm{E} c}\right|^{2}>10^{-5} .
$$

If the E-coplanar optimization obtains the lowest energy but the inequality (A12) is not fulfilled, the ground state is assigned to the $A_{1}$ phase.

\section{Optimizing E-noncoplanar ${ }_{6}$ configuration}


Eq. (45). This can be rewritten as

$$
\begin{gathered}
\mathbf{S}_{0}=\left(\tau_{x}, \tau_{y}, \tau_{z}\right) \\
\mathbf{S}_{1}=\left(-\tau_{x}, \tau_{y}, \tau_{z}\right) \\
\mathbf{S}_{2}=\left(0, t_{a}, t_{b}\right)
\end{gathered}
$$

with $\left(\tau_{x}, \tau_{y}, \tau_{z}\right)$ on the unit sphere and $\left(t_{a}, t_{b}\right)$ on the unit circle

$$
\begin{gathered}
\tau_{x}^{2}+\tau_{y}^{2}+\tau_{z}^{2}=1 \\
t_{a}^{2}+t_{b}^{2}=1
\end{gathered}
$$


Initially, we calculate the energy for $10^{5}$ randomly generated values of $\left(\tau_{x}, \tau_{y}, \tau_{z}\right)$ and $\left(t_{a}, t_{b}\right)$ obeying Eqs. (A16) and (A17). The lowest energy configuration obtained from this random search is then used as input for the iterative minimization step.

In the iterative minimization step, we update the parameters according to the following:

$$
\begin{aligned}
\tau_{\alpha} & \rightarrow \frac{\tau_{\alpha}-c \frac{\partial E}{\partial \tau_{\alpha}}}{\left|\left(\tau_{x}-c \frac{\partial E}{\partial \tau_{x}}, \tau_{y}-c \frac{\partial E}{\partial \tau_{y}}, \tau_{z}-c \frac{\partial E}{\partial \tau_{z}}\right)\right|} \\
t_{\alpha} & \rightarrow \frac{t_{\alpha}-c \frac{\partial E}{\partial t_{\alpha}}}{\left|\left(t_{a}-c \frac{\partial E}{\partial t_{a}}, t_{b}-c \frac{\partial E}{\partial t_{b}}\right)\right|} .
\end{aligned}
$$

The parameter $c$ is initially set to 0.1 . If the update (A18) does not reduce the energy then $c$ is reduced by a factor of 2 and the update is attempted again. This procedure is repeated until the configuration converges.

The set of configurations covered by the E-noncoplanar 6 ansatz (51) includes the $A_{2}$ configurations (when $\mu=-(\kappa-$ $\left.\left.\frac{\pi}{2}\right), v=-\frac{\pi}{6}\right)$. Because of this, if the E-noncoplanar ${ }_{6}$ optimization is found to give the lowest energy of the five possibilities we must check that the obtained configuration has a nonzero value of at least one of the order parameters $\mathbf{m}_{\mathrm{E} \alpha}$. Numerically, we check the condition (A12). If the

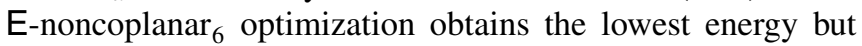
the inequality (A12) is not fulfilled, the ground state is assigned to the $A_{2}$ phase.

\section{Optimizing E-noncoplanar ${ }_{12}$ configuration}

Because the E-noncoplanar ${ }_{12}$ state allows for any configuration of three spins on a single triangle, the configuration space of states is larger and we use simulated annealing rather than a purely random search for the initial optimization, before the iterative minimization step. In the simulated annealing the three spins on a triangle are initialized in a random configuration. Updates are attempted one spin at a time, being certainly accepted if they reduce the energy and accepted with probability $\exp (-\delta E / T)$ if they increase the energy by an amount $\delta E$. Initially, the "temperature," $T=0.2$ in units where $\left|J_{z}\right|=1$ (for Figs. 7-10) or where $J_{x}^{2}+J_{y}^{2}+J_{z}^{2}+D_{y}^{2}+D_{z}^{2}+K^{2}=1$ [for Fig. 11(a)]or where $J_{x}^{2}+J_{y}^{2}+J_{z}^{2}=1$ [for Fig. 11(b)]. The triangle is swept $10^{5}$ times at a given temperature, and the temperature is then reduced by a factor of 0.9 . This procedure is repeated 200 times. There are more than $10^{5}$ sweeps of the triangle with $T=0$, i.e., only accepting energy reducing updates.

The whole annealing procedure is performed from the start three times for each parameter set with the final output being the lowest energy configuration obtained over all three sweeps. To converge the configuration further, there is then an iterative minimization step where each spin component is updated as:

$$
S_{i}^{\alpha} \rightarrow \frac{S_{i}^{\alpha}-c \frac{\partial E}{\partial S_{i}^{\alpha}}}{\left|\left(S_{i}^{x}-c \frac{\partial E}{\partial S_{i}^{x}}, S_{i}^{y}-c \frac{\partial E}{\partial S_{i}^{y}}, S_{i}^{z}-c \frac{\partial E}{\partial S_{i}^{z}}\right)\right|} .
$$

The parameter $c$ is initially set to 0.1 . If the update (A19) does not reduce the energy then $c$ is reduced by a factor of 2 and the update is attempted again. This procedure is repeated until the configuration converges.

If the energy produced from this procedure is lower than the energy produced from optimizing within the $A_{1}, A_{2}, E$ coplanar, or $E$-noncoplanar ${ }_{6}$ phases, then the ground state may be within the $E$-noncoplanar ${ }_{12}$ phase. Because the configuration on the triangle is completely general, to confirm that the configuration has not converged to one of the other phases we check that the inequality (A12) is satisfied and also check that:

$$
\begin{gathered}
m_{\mathrm{A}_{1}}^{2}>10^{-5} \\
m_{\mathrm{A}_{2} \mathrm{a}}^{2}+m_{\mathrm{A}_{2} \mathrm{~b}}^{2}>10^{-5} .
\end{gathered}
$$

If inequalities (A12), (A20), and (A21) are not satisfied, the ground state is assigned to one of the other phases depending on the values of the various $\mathbf{m}_{\gamma}$ (Table I).
[1] A. P. Ramirez, Strongly geometrically frustrated magnets, Annu. Rev. Mater. Sci. 24, 453 (1994).

[2] M. J. Harris, S. T. Bramwell, D. F. McMorrow, T. Zeiske, and K. W. Godfrey, Geometrical Frustration in the Ferromagnetic Pyrochlore $\mathrm{Ho}_{2} \mathrm{Ti}_{2} \mathrm{O}_{7}$, Phys. Rev. Lett. 79, 2554 (1997).

[3] C. L. Henley, Ordering Due to Disorder in a Frustrated Vector Antiferromagnet, Phys. Rev. Lett. 62, 2056 (1989).

[4] Y. Iqbal, T. Müller, P. Ghosh, M. J. P. Gingras, H. O. Jeschke, S. Rachel, J. Reuther, and R. Thomale, Quantum and Classical Phases of the Pyrochlore Heisenberg Model with Competing Interactions, Phys. Rev. X 9, 011005 (2019).

[5] A. Kitaev, Anyons in an exactly solved model and beyond, Ann. Phys. 321, 2 (2006).

[6] I. Rousochatzakis and N. B. Perkins, Classical Spin Liquid Instability Driven By Off-Diagonal Exchange in Strong SpinOrbit Magnets, Phys. Rev. Lett. 118, 147204 (2017).
[7] H. Yan, O. Benton, L. D. C. Jaubert, and N. Shannon, Theory of multiple-phase competition in pyrochlore magnets with anisotropic exchange with application to $\mathrm{Yb}_{2} \mathrm{Ti}_{2} \mathrm{O}_{7}, \mathrm{Er}_{2} \mathrm{Ti}_{2} \mathrm{O}_{7}$, and $\mathrm{Er}_{2} \mathrm{Sn}_{2} \mathrm{O}_{7}$, Phys. Rev. B 95, 094422 (2017).

[8] K. Essafi, O. Benton, and L. D. C. Jaubert, Generic nearestneighbor kagome model: XYZ and Dzyaloshinskii-Moriya couplings with comparison to the pyrochlore-lattice case, Phys. Rev. B 96, 205126 (2017).

[9] Z. Zhu, P. A. Maksimov, S. R. White, and A. L. Chernyshev, Topography of Spin Liquids on a Triangular Lattice, Phys. Rev. Lett. 120, 207203 (2018).

[10] A. Zorko, F. Bert, P. Mendels, K. Marty, and P. Bordet, Ground State of the Easy-Axis Rare-Earth Kagome Langasite $\mathrm{Pr}_{3} \mathrm{Ga}_{5} \mathrm{SiO}_{14}$, Phys. Rev. Lett. 104, 057202 (2010).

[11] S. Ghosh, S. Datta, H. Zhou, M. J. R. Hoch, C. R. Wiebe, P. Schlottmann, and S. Hill, Spin-cluster excitations in the rareearth kagome system $\mathrm{Nd}_{3} \mathrm{Ga}_{5} \mathrm{SiO}_{14}$, Phys. Rev. B 90, 224405 (2014). 
[12] A. Z. Sharma, H. J. Silverstein, A. M. Hallas, G. M. Luke, and C. R. Wiebe, Structure and magnetic properties of new Besubstituted langasites $\mathrm{A}_{3} \mathrm{Ga}_{3} \mathrm{Ge}_{2} \mathrm{BeO}_{14}(\mathrm{~A}=\mathrm{Pr}, \mathrm{Nd}$, and $\mathrm{Sm}), \mathrm{J}$. Solid State Chem. 233, 14 (2016).

[13] M. B. Sanders, K. M. Baroudi, J. W. Krizan, O. A. Mukadam, and R. J. Cava, Synthesis, crystal structure and magnetic properties of novel 2D kagome materials $\mathrm{RE}_{3} \mathrm{Sb}_{3} \mathrm{Mg}_{2} \mathrm{O}_{14}(\mathrm{RE}=$ $\mathrm{La}, \mathrm{Pr}, \mathrm{Sm}, \mathrm{Eu}, \mathrm{Tb}, \mathrm{Ho}$ ): Comparsion to $\mathrm{RE}_{3} \mathrm{Sb}_{3} \mathrm{Zn}_{2} \mathrm{O}_{14}$ family, Phys. Status Solidi B 253, 2056 (2016).

[14] M. B. Sanders, J. W. Krizan, and R. J. Cava, $\mathrm{RE}_{3} \mathrm{Sb}_{3} \mathrm{Zn}_{2} \mathrm{O}_{14}$ $(\mathrm{RE}=\mathrm{La}, \mathrm{Pr}, \mathrm{Nd}, \mathrm{Sm}, \mathrm{Eu}, \mathrm{Gd}):$ A new family of pyrochlore derivatives with rare earth ions on a 2D Kagome lattice, J. Mater. Chem. C 4, 541 (2016).

[15] Z. L. Dun, J. Trinh, K. Li, M. Lee, K. W. Chen, R. Baumbach, Y. F. Hu, Y. X. Wang, E. S. Choi, B. S. Shastry, A. P. Ramirez, and H. D. Zhou, Magnetic Ground States of the Rare-Earth Tripod Kagome Lattice $\mathrm{Mg}_{2} \mathrm{RE}_{3} \mathrm{Sb}_{3} \mathrm{O}_{14}(\mathrm{RE}=\mathrm{Gd}$, Dy, Er), Phys. Rev. Lett. 116, 157201 (2016).

[16] A. Scheie, M. Sanders, J. Krizan, Y. Qiu, R. J. Cava, and C. Broholm, Effective spin- $\frac{1}{2}$ scalar chiral order on kagome lattices in $\mathrm{Nd}_{3} \mathrm{Sb}_{3} \mathrm{Mg}_{2} \mathrm{O}_{14}$, Phys. Rev. B 93, 180407(R) (2016).

[17] Z. L. Dun, J. Trinh, M. Lee, E. S. Choi, K. Li, Y. F. Hu, Y. X. Wang, N. Blanc, A. P. Ramirez, and H. D. Zhou, Structural and magnetic properties of two branches of the tripod-kagomelattice family $A_{2} R_{3} \mathrm{Sb}_{3} \mathrm{O}_{14}(A=\mathrm{Mg}, \mathrm{Zn} ; R=\mathrm{Pr}, \mathrm{Nd}, \mathrm{Gd}, \mathrm{Tb}$, Dy, Ho, Er, Yb), Phys. Rev. B 95, 104439 (2017).

[18] A. Scheie, M. Sanders, J. Krizan, A. D. Christianson, V. O. Garlea, R. J. Cava, and C. Broholm, Crystal field levels and magnetic anisotropy in the kagome compounds $\mathrm{Nd}_{3} \mathrm{Sb}_{3} \mathrm{Mg}_{2} \mathrm{O}_{14}, \mathrm{Nd}_{3} \mathrm{Sb}_{3} \mathrm{Zn}_{2} \mathrm{O}_{14}$, and $\mathrm{Pr}_{3} \mathrm{Sb}_{3} \mathrm{Mg}_{2} \mathrm{O}_{14}$, Phys. Rev. B 98, 134401 (2018).

[19] Z.-F. Ding, Y.-X. Yang, J. Zhang, C. Tan, Z.-H. Zhu, G. Chen, and L. Shu, Possible gapless spin liquid in the rareearth kagome lattice magnet $\mathrm{Tm}_{3} \mathrm{Sb}_{3} \mathrm{Zn}_{2} \mathrm{O}_{14}$, Phys. Rev. B 98, 174404 (2018).

[20] A. Scheie, S. Dasgupta, M. Sanders, A. Sakai, Y. Matsumoto, T. R. Prisk, S. Nakatsuji, R. J. Cava, and C. Broholm, Homogenous reduced moment in a gapful scalar chiral kagome antiferromagnet, Phys. Rev. B 100, 024414 (2019).

[21] M. Elhajal, Benjamin Canals, and C. Lacroix, Symmetry breaking due to Dzyaloshinsky-Moriya interactions in the Kagomé lattice, Phys. Rev. B 66, 014422 (2002).

[22] R. Ballou, B. Canals, M. Elhajal, C. Lacroix, and A.S. Wills, Models for ordering in the jarosites Kagomé systems, J. Magn. Magn. Mater. 262, 465 (2003).

[23] O. Cépas, C. M. Fong, P. W. Leung, and C. Lhuillier, Quantum phase transition induced by Dzyaloshinskii-Moriya interactions in the kagome antiferromagnet, Phys. Rev. B 78, 140405(R) (2008)

[24] L. Messio, O. Cépas, and C. Lhuillier, Schwinger-boson approach to the kagome antiferromagnet with DzyaloshinskiiMoriya interactions: Phase diagram and dynamical structure factors, Phys. Rev. B 81, 064428 (2010).

[25] A. L. Chernyshev and M. E. Zhitomirsky, Quantum Selection of Order in an XXZ Antiferromagnet on a Kagome Lattice, Phys. Rev. Lett. 113, 237202 (2014).

[26] O. Götze and J. Richter, Ground-state phase diagram of the xxz spin-s kagome antiferromagnet: A coupled-cluster study, Phys. Rev. B 91, 104402 (2015).
[27] K. Essafi, O. Benton, and L. D. C. Jaubert, A Kagome map of spin liquids from XXZ to Dzyaloshinskii-Moriya ferromagnet, Nat. Commun. 7, 10297 (2016).

[28] H. J. Changlani, D. Kochkov, K. Kumar, B. K. Clark, and E. Fradkin, Macroscopically Degenerate Exactly Solvable Point in the Spin-1/2 Kagome Quantum Antiferromagnet, Phys. Rev. Lett. 120, 117202 (2018).

[29] K. Morita, M. Kishimoto, and T. Tohyama, Ground-state phase diagram of the kitaev-heisenberg model on a kagome lattice, Phys. Rev. B 98, 134437 (2018).

[30] Yang Yang, Natalia B. Perkins, Fulya Koç, Chi-Huei Lin, and Ioannis Rousochatzakis, Quantum-classical crossover in the spin- $\frac{1}{2}$ heisenberg-kitaev kagome magnet, Phys. Rev. Res. 2, 033217 (2020).

[31] D. Grohol, D. G. Nocera, and D. Papoutsakis, Magnetism of pure iron jarosites, Phys. Rev. B 67, 064401 (2003).

[32] M. Nishiyama, S. Maegawa, T. Inami, and Y. Oka, Magnetic ordering and spin dynamics in potassium jarosite: A Heisenberg kagomé lattice antiferromagnet, Phys. Rev. B 67, 224435 (2003).

[33] T. Morimoto, M. Nishiyama, S. Maegawa, and Y. Oka, Magnetization of new Kagomé lattice antiferromagnets: Cr-Jarosites, $\mathrm{ACr}_{3}(\mathrm{OH})_{6}\left(\mathrm{SO}_{4}\right)_{2}\left[\mathrm{~A}=\mathrm{Na}, \mathrm{K}, \mathrm{Rb}, \mathrm{NH}_{4}\right]$, J. Phys. Soc. Jpn. 72, 2085 (2003).

[34] K. Matan, D. Grohol, D. G. Nocera, T. Yildirim, A. B. Harris, S. H. Lee, S. E. Nagler, and Y. S. Lee, Spin Waves in the Frustrated Kagomé Lattice Antiferromagnet $\mathrm{KFe}_{3}(\mathrm{OH})_{6}\left(\mathrm{SO}_{4}\right)_{2}$, Phys. Rev. Lett. 96, 247201 (2006).

[35] T. Yildirim and A. B. Harris, Magnetic structure and spin waves in the Kagomé jarosite compound $\mathrm{KFe}_{3}\left(\mathrm{SO}_{4}\right)_{2}(\mathrm{OH})_{6}$, Phys. Rev. B 73, 214446 (2006).

[36] R. Okuma, T. Yajima, D. Nishio-Hamane, T. Okubo, and Z. Hiroi, Weak ferromagnetic order breaking the threefold rotational symmetry of the underlying kagome lattice in $\mathrm{CdCu}_{3}(\mathrm{OH})_{6}\left(\mathrm{NO}_{3}\right)_{2} \cdot \mathrm{H}_{2} \mathrm{O}$, Phys. Rev. B 95, 094427 (2017).

[37] S. Onoda and Y. Tanaka, Quantum fluctuations in the effective pseudospin- $\frac{1}{2}$ model for magnetic pyrochlore oxides, Phys. Rev. B 83, 094411 (2011).

[38] G. Chen, Intrinsic transverse field in frustrated quantum ising magnets: Physical origin and quantum effects, Phys. Rev. Res. 1, 033141 (2019).

[39] J. N. Reimers, A. J. Berlinsky, and A.-C. Shi, Mean-field approach to magnetic ordering in highly frustrated pyrochlores, Phys. Rev. B 43, 865 (1991).

[40] G.-B. Sim and S.-B. Lee, Discovery of a new type of magnetic order on pyrochlore spinels, Phys. Rev. B 98, 014423 (2018).

[41] See Supplemental Material http://link.aps.org/supplemental/ 10.1103/PhysRevB.103.174425 for a series of phase diagrams as a function of $J_{x} /\left|J_{z}\right|$ and $J_{y} /\left|J_{z}\right|$, with values of $D_{y} /\left|J_{z}\right|, D_{z} /\left|J_{z}\right|, K /\left|J_{z}\right|$ varying over $\{-0.75,-0.25,0.25,0.75\}$, for both signs of $J_{z}$.

[42] V. Peçanha-Antonio, E. Feng, X. Sun, D. Adroja, H. C. Walker, A. S. Gibbs, F. Orlandi, Y. Su, and T. Brückel, Intermultiplet transitions and magnetic long-range order in Sm-based pyrochlores, Phys. Rev. B 99, 134415 (2019).

[43] M. Maksymenko, V. R. Chandra, and R. Moessner, Classical dipoles on the kagome lattice, Phys. Rev. B 91, 184407 (2015).

[44] C. Wellm, J. Zeisner, A. Alfonsov, M.-I. Sturza, G. Bastien, S. $\mathrm{Gaß,} \mathrm{S.} \mathrm{Wurmehl,} \mathrm{A.} \mathrm{U.} \mathrm{B.} \mathrm{Wolter,} \mathrm{B.} \mathrm{Büchner,} \mathrm{and} \mathrm{V.} \mathrm{Kataev,}$ 
Magnetic interactions in the tripod kagome antiferromagnet $\mathrm{mg}_{2} \mathrm{gd}_{3} \mathrm{sb}_{3} \mathrm{O}_{14}$ probed by static magnetometry and high-field esr spectroscopy, Phys. Rev. B 102, 214414 (2020).

[45] M. E. Brooks-Bartlett, S. T. Banks, L. D. C. Jaubert, A. Harman-Clarke, and P. C. W. Holdsworth, Magnetic-Moment Fragmentation and Monopole Crystallization, Phys. Rev. X 4, 011007 (2014).

[46] J. A. M. Paddison, H. S. Ong, J. O. Hamp, P. Mukherjee, X. Bai, M. G. Tucker, N. P. Butch, C. Castelnovo, M. Mourigal, and S. E. Dutton, Emergent Order in the Kagome Ising Magnet $\mathrm{Dy}_{3} \mathrm{Mg}_{2} \mathrm{Sb}_{3} \mathrm{O}_{14}$, Nat. Commun. 7, 13842 (2016).

[47] G. Möller and R. Moessner, Magnetic multipole analysis of kagome and artificial spin-ice dipolar arrays, Phys. Rev. B 80, 140409(R) (2009).

[48] G.-W. Chern, P. Mellado, and O. Tchernyshyov, Two-Stage Ordering of Spins in Dipolar Spin Ice on the Kagome Lattice, Phys. Rev. Lett. 106, 207202 (2011).
[49] S. A. Owerre, Topological thermal hall effect in frustrated kagome antiferromagnets, Phys. Rev. B 95, 014422 (2017).

[50] R. Seshadri and D. Sen, Topological magnons in a kagome-lattice spin system with $X X Z$ and Dzyaloshinskii-Moriya interactions, Phys. Rev. B 97, 134411 (2018).

[51] A. Mook, J. Henk, and I. Mertig, Thermal Hall effect in noncollinear coplanar insulating antiferromagnets, Phys. Rev. B 99, 014427 (2019).

[52] G. Palle and O. Benton, Exactly solvable spin-1/2 xyz models with highly-degenerate, partially ordered, ground states, arXiv:2101.12140.

[53] O. Benton, L. D. C. Jaubert, H. Yan, and N. Shannon, A spin-liquid with pinch-line singularities on the pyrochlore lattice, Nat. Commun. 7, 11572 (2016). 